\title{
Comparison of viscosity solutions of fully nonlinear degenerate parabolic Path-dependent PDEs
}

\author{
Zhenjie REN* Nizar TouzI ${ }^{\dagger} \quad$ Jianfeng ZHANG ${ }^{\ddagger}$ \\ January 15, 2018
}

\begin{abstract}
We prove a comparison result for viscosity solutions of (possibly degenerate) parabolic fully nonlinear path-dependent PDEs. In contrast with the previous result in Ekren, Touzi \& Zhang [1], our conditions are easier to check and allow for the degenerate case, thus including first order path-dependent PDEs. Our argument follows the regularization method as introduced by Jensen, Lions \& Souganidis [12] in the corresponding finite-dimensional PDE setting. The present argument significantly simplifies the comparison proof in [1], but requires an $\mathbb{L}^{p}$-type of continuity (with respect to the path) for the viscosity semi-solutions and for the nonlinearity defining the equation.
\end{abstract}

\section{Introduction}

This paper provides a proof for the comparison result for viscosity solutions of the fully nonlinear path dependent partial differential equation:

$$
-\partial_{t} u(t, \omega)-G\left(t, \omega, u(t, \omega), \partial_{\omega} u(t, \omega), \partial_{\omega \omega}^{2} u(t, \omega)\right) \leq 0 \quad \text { on } \quad[0, T) \times \Omega .
$$

Here, $T>0$ is a given terminal time, and $\omega \in \Omega$ is a continuous path from $[0, T]$ to $\mathbb{R}^{d}$ starting from the origin. The nonlinearity $G$ is a mapping from $[0, T] \times \Omega \times \mathbb{R} \times \mathbb{R}^{d} \times \mathbb{S}^{d}$ to $\mathbb{R}$, where $\mathbb{S}^{d}$ denotes the set of all $d \times d$-symmetric matrices.

Such equations arise naturally in many applications. For instance, the dynamic programming equation (also called Hamilton-Jacobi-Bellman equation) associated to a problem of stochastic control of non-Markov diffusions falls in the class of equations (1.1), see [10. In particular hereditary control problems may be addressed in this context rather than embedding the problem into a PDE

\footnotetext{
*CMAP, Ecole Polytechnique Paris, ren@cmap.polytechnique.fr.

$\dagger^{\dagger}$ MAP, Ecole Polytechnique Paris, nizar.touzi@polytechnique.edu. Research supported by ANR the Chair Financial Risks of the Risk Foundation sponsored by Société Générale, and the Chair Finance and Sustainable Development sponsored by EDF and Calyon.

${ }^{\ddagger}$ University of Southern California, Department of Mathematics, jianfenz@usc.edu. Research supported in part by NSF grant DMS 1413717.
} 
on the Hilbert space $\mathbb{L}^{2}([0, T]) \supset \Omega$, see [6]. Similarly, stochastic differential games with non-Markov controlled dynamics lead to path-dependent Isaac-Hamilton-Jacobi-Bellman equations of the form (1.1), see [17. The notion of nonlinear path dependent partial differential equation was first proposed by Peng [15]. A crucial tool to study such equation is the functional Itô calculus, initiated by Dupire [7, and further studied by Cont \& Fournie [3]. We also refer to Peng \& Wang [16] for some study on classical solutions of semilinear equations.

The notion of viscosity solutions studied in this paper was introduced by Ekren, Keller, Touzi \& Zhang [8] in the semilinear context, and further extended to the fully nonlinear case by Ekren, Touzi \& Zhang [10, 11]. Following the lines of the classical Crandall \& Lions [5 notion of viscosity solutions, supersolutions and subsolutions are defined through tangent test functions. However, while Crandall \& Lions consider pointwise tangent functions, the tangency conditions in the present path-dependent setting is in the sense of the mean with respect to an appropriate class of probability measures $\mathcal{P}$. In particular, when restricted to the Markovian case, our notion of viscosity solutions involves a larger set of test functions. This is in favor of uniqueness but may make the existence issue more difficult. We refer to Ren, Touzi \& Zhang [19] for an overview.

Throughout this paper, the notion of $\mathcal{P}$-viscosity solution refers that introduced in 8 , 10]. For the sake of clarity, the classical notion of viscosity solutions based on pointwise tangent test functions will be sometimes referred to as the Crandall-Lions notion of viscosity solution.

The wellposedness of the notion of $\mathcal{P}$-viscosity solutions was first proved in $[8$ ] in the semilinear case, and later extended to the fully nonlinear case in [11. In contrast with the classical wellposedness theory for the Crandall-Lions viscosity solutions in finite dimensional spaces, the comparison and existence results proved in [8, 11] are inter-connected. Moreover, the proof relies heavily on the corresponding finite-dimensional PDE results applied to path-frozen versions of (1.1), and thus does not really take advatange of the larger class of test functions. Finally, the technical conditions of [8, 11] exclude the degenerate case. In particular, their main wellposedness result can not be viewed as an extension of Lukoyanov [14, where the author studied the wellposedness of the viscosity solutions to the first order path-dependent PDEs.

In our recent paper [21] we provided a purely probabilistic comparison proof in the semilinear setting, which is completely disconnected from the existence result and which allows for degenerate equations. The importance of a separate proof of comparison was highlighted in the Crandall-Lions theory of viscosity solutions: it allows access to the Perron existence argument, and was shown to play an important role in the regularity of viscosity solutions, and in the convergence of numerical approximations together with the analysis of the order of the corresponding error. Similar to the classical finite-dimensional theory of viscosity solutions, the (disconnected) comparison result in [21] opens the door for an existence argument by the so-called Perron method, see Ren [18, or by a limiting argument à la Barles \& Souganidis [1, see Zhang \& Zhuo [22] and Ren \& Tan [20].

Our argument in 21] was crucially based on an adaptation of the Caffarelli \& Cabre 2] notion of punctual differentiation to our setting, namely the $\mathcal{P}$-punctual differentiation. In particular, denoting by $\mathbb{P}_{0}$ the Wiener measure on the space of continuous paths, we have reported in [21] 
an easy proof of the equivalence between our notion of $\left\{\mathbb{P}_{0}\right\}$-viscosity subsolution of the heat equation and the submartingale property. This equivalence implies an easy proof of the comparison result for linear path-dependent PDEs, thus highlighting the importance of enlarging the set of test functions under the notion of $\left\{\mathbb{P}_{0}\right\}$-viscosity solutions. The semilinear case is more involved, but uses standard stochastic analysis arguments. A crucial (and surprising) result obtained in [21] is that all $\mathcal{P}$-viscosity subsolutions with appropriate integrability are $\mathcal{P}$-punctually differentiable Leb $\otimes \mathbb{P}_{0}-$ almost everywhere on $[0, T] \times \Omega$. We recall that in the finite-dimensional context, the punctual differentiability is satisfied by an appropriate approximation of the subsolution.

The main contribution of this paper is to provide a comparison result for the viscosity solutions of fully nonlinear path-dependent PDEs (1.1) which does not involve the existence issue. Our result is established under general conditions on the nonlinearity. Namely, we establish the comparison between $d_{p}$-uniformly continuous subsolutions and supersolutions under the conditions that the nonlinearity $G$ is

- $d_{p}$-uniformly continuous in $\theta$, uniformly in $(y, z, \gamma)$,

- and Lipschitz-continuous in $(y, z, \gamma)$, uniformly in $\theta$.

The last conditions represent a significant simplification of the assumptions required in Ekren, Touzi \& Zhang [11. Moreover, we emphasize that our conditions allow for degenerate parabolic equations, and therefore contain the first order path-dependent Hamilton-Jacobi equations of Lukoyanov [14.

Our arguments are inspired from the work of Jensen, Lions \& Souganidis [12, in which one of the main ideas is to find approximations of viscosity sub- and supersolutions of PDEs. The approximations proposed in [12] are due to Lasry \& Lions [13]. Let $u$ be a viscosity subsolution, and $u^{n}$ be the approximations. A careful examination of their proof shows that good approximations should in general satisfy:

(A1) $\lim _{n \rightarrow \infty} u^{n}=u$;

(A2) $u^{n}$ are more regular than $u$ (thus we may call the approximations as regularization);

(A3) $u^{n}$ are still viscosity subsolutions for some equations approximating the original one.

A similar regularization was introduced in Ren [18, for functions in the path space, in order to study the comparison of semi-continuous viscosity solutions to semilinear path-dependent PDEs. However such a regularization fails to achieve the purpose of the present paper.

As a key technical tool in this paper, we introduce a new regularization for viscosity sub- and supersolutions in the context of fully nonlinear path-dependent PDEs, which allows to prove the final comparison result. Constrained by our method, we are unfortunately not able to compare the viscosity sub- and supersolutions which are continuous in the (pseudo-)distance $d_{\infty}$,

$$
d_{\infty}\left((t, \omega),\left(t^{\prime}, \omega^{\prime}\right)\right)=\left|t-t^{\prime}\right|+\left\|\omega_{t \wedge \cdot}-\omega_{t^{\prime} \wedge \cdot}^{\prime}\right\|_{\infty},
$$

used in the previous works on path dependent PDEs. Instead, we prove the comparison for viscosity sub- and supersolutions which are continuous in the sense of the following (pseudo-)distance:

$$
d_{p}\left((t, \omega),\left(t^{\prime}, \omega^{\prime}\right)\right)=\left|t-t^{\prime}\right|+\left\|\omega_{t \wedge} \cdot-\omega_{t^{\prime} \wedge}^{\prime} \cdot\right\|_{p}^{p}, \quad \text { where }\|\omega\|_{p}^{p}=\int_{0}^{T+1}\left|\omega_{t}\right|^{p} d t .
$$


This continuity is slightly stronger than that under $d_{\infty}$. However, since $\|\omega\|_{p} \rightarrow\|\omega\|_{\infty}$, our continuity requirement can be viewed as a slight strengthening of the $d_{\infty}$-continuity. In order to justify the relevance of the $d_{p}$-continuity, we provide in this paper a large class of path-dependent fully nonlinear equations with unique $d_{p}$-continuous viscosity solutions. This is achieved by complementing our comparison result with an example of stochastic control problem whose value function is a $d_{p}$-continuous $\mathcal{P}$-viscosity solution of the corresponding path-dependent dynamic programming equation.

Given a $\mathcal{P}$-viscosity solution $u$, the regularization introduced in the present paper defines functions $u^{n}$ satisfying the above requirement (A1). However, rather than verifying (A2) and (A3) in the sense of $\mathcal{P}$-viscosity solutions, we show that $u^{n}$ induces a continuous finite-dimensional function which is a viscosity solution of an appropriate PDE in the classical sense of Crandall-Lions. We recall that this is a weaker conclusion than the corresponding notion of $\mathcal{P}$-viscosity solutions. This allows to reduce the comparison task to the well-established notion of viscosity solutions in the finite-dimensional context, and represents the major difference with the approach used in our previous paper 21] focused on the semilinear case.

The rest of the paper is organized as follows. Section 2 introduces the main notations. Section 3 recalls some useful results from the previous work on path dependent PDEs. Section 4 states the main assumptions and results of this paper. In Section 5 we introduce the regularization, and prove its main properties. Further, in Section 6 we use the regularization to prove the comparison result. Finally, Section 7 concludes the paper by an example of a stochastic control problem whose value function is a $d_{p}$-continuous $\mathcal{P}$-viscosity solution of the corresponding path-dependent dynamic programming equation, under natural assumptions on the ingredients of the control problem.

\section{Notations}

Throughout this paper let $T>0$ be a given finite maturity, $\Omega:=\left\{\omega \in C\left([0, T] ; \mathbb{R}^{d}\right): \omega_{0}=0\right\}$ be the set of continuous paths starting from the origin, and $\Theta:=[0, T] \times \Omega$. For the convenience of notation, we often denote by $\theta$ the pair $(t, \omega)$. We denote by $B$ the canonical process on $\Omega$, by $\mathbb{F}=\left\{\mathcal{F}_{t}, 0 \leq t \leq T\right\}$ the canonical filtration, by $\mathbb{P}_{0}$ the Wiener measure on $\Omega$, and by $\mathcal{T}$ the set of all $\mathbb{F}$-stopping times taking values in $[0, T]$. Further, for $\mathrm{H} \in \mathcal{T}$, denote by $\mathcal{T}_{\mathrm{H}}$ the subset of $\tau \in \mathcal{T}$ taking values in $[0, \mathrm{H}]$.

For $\omega, \omega^{\prime} \in \Omega$ and $t \in[0, T]$, we define

$$
\left(\omega \otimes_{t} \omega^{\prime}\right)_{s}:=\omega_{s} \mathbf{1}_{\{s<t\}}+\left(\omega_{t}+\omega_{s-t}^{\prime}\right) \mathbf{1}_{\{s \geq t\}} .
$$

Let $\xi: \Omega \rightarrow \mathbb{R}$ be $\mathcal{F}_{T}$-measurable random variable. For any $\theta=(t, \omega) \in \Theta$, define

$$
\xi^{\theta}\left(\omega^{\prime}\right):=\xi\left(\omega \otimes_{t} \omega^{\prime}\right) \quad \text { for all } \quad \omega^{\prime} \in \Omega .
$$

Clearly, $\xi^{\theta}$ is $\mathcal{F}_{T-t}$-measurable, and thus $\mathcal{F}_{T}$-measurable. Similarly, given a process $X$ defined on $\Omega$, we denote:

$$
X_{s}^{\theta}\left(\omega^{\prime}\right):=X_{t+s}\left(\omega \otimes_{t} \omega^{\prime}\right), \text { for } s \in[0, T-t], \omega^{\prime} \in \Omega
$$


Clearly, if $X$ is $\mathbb{F}$-adapted, then so is $X^{\theta}$.

As in Ekren, Touzi \& Zhang [11, for every constant $L>0$, we denote by $\mathcal{P}_{L}$ the collection of all continuous semimartingale measures $\mathbb{P}$ on $\Omega$ whose drift and diffusion are bounded by constant $L$, respectively. More precisely, let $\tilde{\Omega}:=\Omega \times \Omega \times \Omega$ be an enlarged canonical space, $\tilde{B}:=(B, A, M)$ be the canonical process. A probability measure $\mathbb{P} \in \mathcal{P}_{L}$ means that there exists an extension $\mathbb{Q}^{\alpha, \beta}$ of $\mathbb{P}$ on $\tilde{\Omega}$ such that:

$$
\begin{aligned}
& B=A+M, \quad A \text { is absolutely continuous, } M \text { is a martingale, } \quad \mathbb{Q}^{\alpha, \beta} \text {-a.s. } \\
& \left\|\alpha^{\mathbb{P}}\right\|_{\infty},\left\|\beta^{\mathbb{P}}\right\|_{\infty} \leq L, \quad \text { where } \alpha_{t}^{\mathbb{P}}:=\frac{d A_{t}}{d t}, \beta_{t}^{\mathbb{P}}:=\sqrt{\frac{d\langle M\rangle_{t}}{d t}},
\end{aligned}
$$

We also introduce the sublinear and superlinear expectation operators associated to $\mathcal{P}_{L}$ :

$$
\overline{\mathcal{E}}_{L}:=\sup _{\mathbb{P} \in \mathcal{P}_{L}} \mathbb{E}^{\mathbb{P}} \quad \text { and } \quad \underline{\mathcal{E}}_{L}:=\inf _{\mathbb{P} \in \mathcal{P}_{L}} \mathbb{E}^{\mathbb{P}}
$$

One may easily prove the following lemma.

Lemma 2.1 There is a constant $C>0$ such that we have for all $\mathbb{P} \in \mathcal{P}_{L}$ and $\mathrm{H} \in \mathcal{T}$ that

$$
\left|\mathbb{E}^{\mathbb{P}}\left[B_{\mathrm{H}}\right]\right| \leq C \mathbb{E}^{\mathbb{P}}[\mathrm{H}] \quad \text { and } \quad \mathbb{E}^{\mathbb{P}}\left[\left|B_{\mathrm{H}}\right|^{2}\right] \leq C \mathbb{E}^{\mathbb{P}}[\mathrm{H}] .
$$

In this paper, we consider a new $\mathbb{L}^{p}$-type of distance in the space $\Theta$.

Definition 2.2 For $p \geq 1$, we introduce the following distance for the space $\Theta$ :

$$
d_{p}\left(\theta, \theta^{\prime}\right):=\left|t-t^{\prime}\right|+\left\|\omega_{t \wedge} \cdot-\omega_{t^{\prime} \wedge}^{\prime} \cdot\right\|_{p}, \quad \text { for all } \theta, \theta^{\prime} \in \Theta
$$

where

$$
\|\omega\|_{p}^{p}:=\left|\omega_{T}\right|^{p}+\int_{0}^{T}\left|\omega_{s}\right|^{p} d s, \quad \text { for all } \omega, \omega^{\prime} \in \Omega
$$

We say that a function $f: \Theta \rightarrow \mathbb{R}$ is $d_{p}$-continuous, if $f$ is continuous with respect to $d_{p}(\cdot, \cdot)$.

Remark 2.3 Let $d_{\infty}(\cdot, \cdot)$ be the distance between continuous paths introduced by Dupire [7, i.e.

$$
d_{\infty}\left(\theta, \theta^{\prime}\right):=\left|t-t^{\prime}\right|+\max _{0 \leq s \leq T}\left|\omega_{s \wedge t}-\omega_{s \wedge t^{\prime}}^{\prime}\right| .
$$

Note that

$$
d_{p}\left(\theta, \theta^{\prime}\right) \leq C d_{\infty}\left(\theta, \theta^{\prime}\right) \text { and } \lim _{p \rightarrow \infty} d_{p}\left(\theta, \theta^{\prime}\right)=d_{\infty}\left(\theta, \theta^{\prime}\right) .
$$

In particular, a $d_{p}$-continuous function is automatically continuous in Dupire's sense.

For later use, we observe that

$$
\left\|\omega_{t \wedge} \cdot\right\|_{p}^{p}=\int_{0}^{T+1}\left|\omega_{t \wedge s}\right|^{p} d s=(T+1-t)\left|\omega_{t}\right|^{p}+\int_{0}^{t}\left|\omega_{s}\right|^{p} d s .
$$

Example 7.1 in Appendix provides sufficient conditions for the value function of a stochastic control problem to be uniformly $d_{p}$-continuous. On the other hand, as discussed in Ekren, Touzi 
\& Zhang [10, the value function of a stochastic control problem can be proved to be a viscosity solution of the corresponding path dependent Hamilton-Jaccobi-Bellman equation. Therefore, there are many examples of fully nonlinear path dependent PDEs which have uniformly $d_{p}$-continuous viscosity solutions. In this paper, we focus on the uniqueness of such solutions.

We would like to emphasize that, throughout this paper, $C$ denotes a generic constant, which may change from line to line. For example the reader may find $2 C \leq C$, without any contradiction as the left-hand side $C$ is different from the right-hand side $C$.

\section{Preliminaries}

Consider the fully nonlinear parabolic PDE:

$$
-\partial_{t} u-g\left(t, x, u, D u, D^{2} u\right)=0 \quad t<T, \quad x \in \mathbb{R}^{d},
$$

where $\partial_{t}$ denotes the time derivative, and $D u, D^{2} u$ denote the space gradient and Hessian, respectively.

We first recall the definition of the classical Crandall-Lions viscosity solutions for parabolic PDEs. For $\alpha \in \mathbb{R}, \beta \in \mathbb{R}^{d}, \gamma \in \mathbb{S}^{d}$, define the paraboloid $\psi^{\alpha, \beta, \gamma}$ :

$$
\psi^{\alpha, \beta, \gamma}(t, x):=\alpha t+\beta \cdot x+\frac{1}{2} x^{\mathrm{T}} \gamma x, \quad t \geq 0, x \in \mathbb{R}^{d} .
$$

Then define the jets:

$$
\begin{aligned}
& \underline{J} u(t, x):=\left\{(\alpha, \beta, \gamma): \quad u(t, x) \geq u(s, y)-\psi^{\alpha, \beta, \gamma}(s-t, y-x)+o\left(|s-t|,|y-x|^{2}\right)\right\} \\
& \bar{J} v(t, x):=\left\{(\alpha, \beta, \gamma): \quad v(t, x) \leq v(s, y)-\psi^{\alpha, \beta, \gamma}(s-t, y-x)+o\left(|s-t|,|y-x|^{2}\right)\right\} .
\end{aligned}
$$

We say that function $u$ is a viscosity subsolution of the PDE (3.1), if

$$
-\alpha-g(t, x, u, \beta, \gamma) \leq 0, \quad \text { for all }(t, x) \in(0, T) \times \mathbb{R}^{d} \text { and }(\alpha, \beta, \gamma) \in \underline{J} u(t, x) .
$$

Similarly, a function $v$ is a viscosity supersolution of the PDE (3.1), if

$$
-\alpha-g(t, x, v, \beta, \gamma) \geq 0, \quad \text { for all }(t, x) \in(0, T) \times \mathbb{R}^{d} \text { and }(\alpha, \beta, \gamma) \in \bar{J} v(t, x) .
$$

In this paper, we consider the fully nonlinear parabolic path dependent PDE:

$$
-\partial_{t} u-G\left(\theta, u, \partial_{\omega} u, \partial_{\omega \omega}^{2} u\right)=0 .
$$

In our previous work [19, 21] on viscosity solutions of path-depedent PDEs, it was already understood that one can define viscosity solutions via jets. For simplicity, this paper starts directly from this definition as it avoids to introduce the notion of smooth processes (i.e. those processes which satisfy an Itô formula simultaneously under all probability measures $\left.\mathbb{P} \in \mathcal{P}_{L}\right)$. Let

$$
\phi^{\alpha, \beta, \gamma}(\theta):=\alpha t+\beta \cdot \omega_{t}+\frac{1}{2} \omega_{t}^{\mathrm{T}} \gamma \omega_{t}, \quad \theta \in \Theta,
$$


for some $(\alpha, \beta, \gamma) \in \mathbb{R} \times \mathbb{R}^{d} \times \mathbb{S}_{d}$. We then introduce the corresponding subjet and superjet:

$$
\begin{aligned}
\underline{\mathcal{J}}_{L} u(\theta) & :=\left\{(\alpha, \beta, \gamma) \in \mathbb{R} \times \mathbb{R}^{d} \times \mathbb{S}_{d}: u(\theta)=\max _{\tau \in \mathcal{T}_{\mathcal{H}_{\delta}}} \overline{\mathcal{E}}_{L}\left[\left(u^{\theta}-\phi^{\alpha, \beta, \gamma}\right)(\tau, B)\right], \text { for some } \delta>0\right\}, \\
\overline{\mathcal{J}}_{L} v(\theta) & :=\left\{(\alpha, \beta, \gamma) \in \mathbb{R} \times \mathbb{R}^{d} \times \mathbb{S}_{d}: v(\theta)=\min _{\tau \in \mathcal{T}_{\mathcal{H}_{\delta}}} \underline{\mathcal{E}}_{L}\left[\left(v^{\theta}-\phi^{\alpha, \beta, \gamma}\right)(\tau, B)\right], \text { for some } \delta>0\right\},
\end{aligned}
$$

where

$$
\mathrm{H}_{\delta}:=\delta \wedge \inf \left\{t \geq 0:\left|B_{t}\right| \geq \delta\right\}
$$

is a stopping time, and is called the localization.

Definition 3.1 A function $u:[0, T] \times \Omega \longrightarrow \mathbb{R}$ is a

- $\mathcal{P}_{L}$-viscosity subsolution of (3.2) if $-\alpha-G(\theta, u(\theta), \beta, \gamma) \leq 0$ for all $\theta \in \Theta,(\alpha, \beta, \gamma) \in \underline{\mathcal{J}}_{L} u(\theta)$;

- $\mathcal{P}_{L}$-viscosity supersolution of (3.2) if $-\alpha-G(\theta, u(\theta), \beta, \gamma) \geq 0$ for all $\theta \in \Theta,(\alpha, \beta, \gamma) \in \overline{\mathcal{J}}_{L} u(\theta)$.

It was proved in Ren, Touzi \& Zhang [19] that this definition is equivalent to the original definition of Ekren, Touzi \& Zhang [10] whenever the function $u$ and the nonlinearity $G(., y, z, \gamma)$ are $d_{\infty}$-continuous. By following the same line of argument, the same equivalence of definitions holds under our $d_{p}$-continuity assumptions.

We next recall the Snell envelop characterization of the optimal stopping problem under nonlinear expectation, see Theorem 3.5 in Ekren, Touzi \& Zhang [9].

Lemma 3.2 Let $X: \Theta \rightarrow \mathbb{R}$ be $d_{p}$-uniformly continuous. Consider the optimal stopping problem:

$$
V(\theta):=\sup _{\tau \in \mathcal{T}_{\text {H }_{\delta}^{\theta}-t}} \overline{\mathcal{E}}_{L}\left[X_{\tau}^{\theta}\right]
$$

Then, denoting $\hat{V}_{t}:=V_{t} 1_{\left\{t<\mathrm{H}_{\delta}\right\}}+V_{\mathrm{H}_{\delta}-} 1_{\left\{t \geq \mathrm{H}_{\delta}\right\}}$, we have

$$
V_{0}=\overline{\mathcal{E}}_{L}\left[X_{\tau^{*}}\right] \text { where } \tau^{*}:=\inf \left\{t \geq 0: X_{t}=\hat{V}_{t}\right\} .
$$

As a consequence of the last fundamental result from optimal stopping theory, we now provide our main technical substitute for the local compactness argument in the finite-dimensional CrandallLions viscosity solutions.

Lemma 3.3 Let $u$ be $d_{p}$-uniformly continuous function satisfying $u(\mathbf{0})>\overline{\mathcal{E}}_{L}\left[\left(u-\phi^{\alpha, \beta, \gamma}\right)\left(\mathrm{H}_{\delta}, B\right)\right]$, for some $\delta>0$ and $(\alpha, \beta, \gamma) \in \mathbb{R} \times \mathbb{R}^{d} \times \mathbb{S}^{d}$. Then, there exists $\theta^{*}=\left(t^{*}, \omega^{*}\right)$ such that

$$
t^{*}<\mathrm{H}_{\delta}\left(\omega^{*}\right) \quad \text { and } \quad\left(\alpha, \beta+\gamma \omega_{t^{*}}^{*}, \gamma\right) \in \underline{\mathcal{J}}_{L} u\left(\theta^{*}\right) .
$$

Proof Define the optimal stopping problem $V$ :

$$
V(\theta):=\sup _{\tau \in \mathcal{T}_{\text {H }_{\delta}-t}} \overline{\mathcal{E}}_{L}\left[X^{\theta}(\tau, B)\right] .
$$

with $X:=u-\phi^{\alpha, \beta, \gamma}$. Let $\tau^{*} \in \mathcal{T}_{\mathrm{H}_{\delta}}$ be the optimal stopping rule. By Lemma 3.2 we have

$$
\overline{\mathcal{E}}_{L}\left[X_{\tau^{*}}\right]=V_{0} \geq X_{0}>\overline{\mathcal{E}}_{L}\left[X_{\mathrm{H}_{\delta}}\right] \text { and } X_{\tau^{*}}=\hat{V}_{\tau^{*}},
$$


So there exists $\omega^{*} \in \Omega$ such that $t^{*}:=\tau^{*}\left(\omega^{*}\right)<\mathrm{H}_{\delta}\left(\omega^{*}\right)$ and $X_{t^{*}}\left(\omega^{*}\right)=\hat{V}_{t^{*}}\left(\omega^{*}\right)=V_{t^{*}}\left(\omega^{*}\right)$, i.e.

$$
\begin{aligned}
u\left(\theta^{*}\right) & =\sup _{\tau \in \mathcal{T}_{\mathrm{H}_{\delta}^{* *}-t^{*}}} \overline{\mathcal{E}}_{L}\left[u_{\tau}^{\theta^{*}}-\alpha \tau-\beta \cdot B_{\tau}-\frac{1}{2}\left(\omega_{t^{*}}^{*}+B_{\tau}\right)^{\mathrm{T}} \gamma\left(\omega_{t^{*}}^{*}+B_{\tau}\right)+\frac{1}{2}\left(\omega_{t^{*}}^{*}\right)^{\mathrm{T}} \gamma \omega_{t^{*}}^{*}\right] \\
& =\sup _{\tau \in \mathcal{T}_{\mathrm{H}_{\delta}^{* *}-t^{*}}} \overline{\mathcal{E}}_{L}\left[u_{\tau}^{\theta^{*}}-\alpha \tau-\left(\beta+\gamma \omega_{t^{*}}^{*}\right) \cdot B_{\tau}-\frac{1}{2} B_{\tau}^{\mathrm{T}} \gamma B_{\tau}\right]
\end{aligned}
$$

By the definition of $\underline{\mathcal{I}}_{L} u$, this means that $\left(t^{*}, \omega^{*}\right)$ is the required point.

\section{Main result}

We shall establish our main comparison result under the following general conditions on the nonlinearity $G$.

Assumption 4.1 The nonlinearity $G$ satisfies the following conditions:

(i) $G$ is elliptic, i.e. $G(\theta, y, z, \gamma) \leq G\left(\theta, y, z, \gamma^{\prime}\right)$, for $\gamma \leq \gamma^{\prime}$.

(ii) $G$ is $d_{p}$-uniformly continuous in $\theta$, uniformly in $(y, z, \gamma)$, i.e. for some continuity modulus $\rho^{G}$ :

$$
\left|G(\theta, y, z, \gamma)-G\left(\theta^{\prime}, y, z, \gamma\right)\right| \leq \rho^{G}\left(d_{p}\left(\theta, \theta^{\prime}\right)\right), \quad \text { for all } \quad(y, z, \gamma) \in \mathbb{R} \times \mathbb{R}^{d} \times \mathbb{S}^{d} .
$$

(iii) $G$ is uniformly Lipschitz continuous in $(y, z, \gamma)$, i.e. there is a constant $L_{0}$ such that

$$
\left|G(\theta, y, z, \gamma)-G\left(\theta, y^{\prime}, z^{\prime}, \gamma^{\prime}\right)\right| \leq L_{0}\left(\left|y-y^{\prime}\right|+\left|z-z^{\prime}\right|+\left|\gamma-\gamma^{\prime}\right|\right), \text { for all } \theta \in \Theta .
$$

The first condition restricts the path-dependent PDE to the parabolic case. The remaining technical conditions are required in our proofs. In contrast with the comparison result established in Ekren, Touzi \& Zhang [11, we emphasize that the above conditions do not exclude degenerate path-dependent second order PDEs. In particular, our main result, Theorem 4.2 below, holds for first order path-dependent PDEs which satisfy the above conditions (ii)-(iii), and thus covers the path-dependent Hamilton-Jacobi PDEs analyzed in Lukoyanov [14.

Theorem 4.2 Let $u, v: \Theta \rightarrow \mathbb{R}$ be bounded and $d_{p}$-uniformly continuous $\mathcal{P}_{L}$-viscosity subsolution and $\mathcal{P}_{L}$-viscosity supersolution of (3.2), respectively. Under Assumption [4.1. if $u(T, \cdot) \leq v(T, \cdot)$, then $u \leq v$ on $\Theta$.

The proof of this result will be provided in Section 6 after the preparations of Section 5 We conclude this short section by some remarks which will be recalled in our subsequent proof of Theorem 4.2

Remark 4.3 If $u$ is a $\mathcal{P}_{L}$-viscosity subsolution of (3.2), then function $\tilde{u}:=e^{-L t} u$ is a $\mathcal{P}_{L}$-viscosity subsolution of

$$
-\partial_{t} \tilde{u}-\tilde{G}\left(\theta, \tilde{u}, \partial_{\omega} \tilde{u}, \partial_{\omega \omega}^{2} \tilde{u}\right)=0 .
$$

where $\tilde{G}$ is non-decreasing in $y$. A similar statement holds for $\mathcal{P}_{L}$-viscosity supersolutions. 
In view of this result, , we shall assume throughout the paper, without loss of generality, that nonlinearity $G$ is non-decreasing in $y$. Consequently, we may modify Assumption 4.1 (iii) as:

(iii') There is a constant $L_{0}$ such that

$$
G(\theta, y, z, \gamma)-G\left(\theta, y^{\prime}, z^{\prime}, \gamma^{\prime}\right) \leq L_{0}\left(\left(y-y^{\prime}\right)^{+}+\left|z-z^{\prime}\right|+\left|\gamma-\gamma^{\prime}\right|\right), \quad \text { for all } \quad \theta \in \Theta .
$$

Remark 4.4 Since all $d_{p}$-uniformly continuous function is $d_{q}$-uniformly continuous, for $q>p$, it is sufficient to prove the theorem for the largest possible values of $p$. For technical reasons, we shall choose $p$ to be odd and $p>1$.

\section{Regularization}

In this section, we introduce the crucial regularization $u^{n}$ of the viscosity subsolution $u$. Recall the $\|\cdot\|_{p}$-norm defined in (2.2). For $s \geq 0$, a càdlàg path $\eta$, and any increasing function $\ell$, we define the penalization function:

$$
\Phi(s, \eta, \theta, \ell):=\|\ell-I\|_{\infty}^{\frac{2}{3 p+3}}+\left\|\eta_{s \wedge \ell(\cdot)}-\omega_{t \wedge} \cdot\right\|_{p+1}^{p+1}, \quad \theta \in \Theta
$$

where $I:[0, T] \rightarrow[0, T]$ is the identity function and $\|\ell-I\|_{\infty}:=\sup _{0 \leq t \leq T}|\ell(t)-t|$. Denote $\mathbf{0}:=(0,0)$, and define the regularization:

$$
u^{n}(s, \eta):=\sup _{\theta \in \Theta \backslash \mathbf{0}, \ell \in \mathcal{L}_{t, s}}\{u(\theta)-n \Phi(s, \eta, \theta, \ell)\},
$$

where, for $s>0, \mathcal{L}_{t, s}$ is the set of the increasing functions $\ell:[0, T] \rightarrow \mathbb{R}$ such that

$$
\left.\ell\right|_{[0, t]}:[0, t] \rightarrow[0, s] \text { is an increasing bijection, and } \ell(r):=r-t+s \text { for } r \in(t, T],
$$

For $s=0$, this set is reduced to a signleton $\mathcal{L}_{t, 0}=\left\{\ell^{t, 0}\right\}$, with $\ell^{t, 0}:[0, T] \rightarrow \mathbb{R}$ defined as:

$$
\ell^{t, 0}(r)=(r-t) 1_{(t, T]}(r), \quad \text { for all } r \in[0, T] .
$$

Notice that for $s>0$, any $\ell \in \mathcal{L}_{t, s}$ is an injection, so that we can naturally define the inverse function $\ell^{-1}$ on the image of $\ell$. For later use, we also note that for all $\ell \in \mathcal{L}_{t, s}$, we have

$$
\left\|\eta_{s \wedge \ell(\cdot)}-\omega_{t \wedge \cdot}\right\|_{p+1}=\left\|\eta_{\ell(t \wedge \cdot)}-\omega_{t \wedge \cdot}\right\|_{p+1} .
$$

Remark 5.1 Notice from (5.2), (5.3) and (5.4) that the values of the function $\ell$ on $(t, T]$ do not have any impact on the value of the penalization $\Phi(s, \eta, \theta, \ell)$. In order to construct a function $\ell \in \mathcal{L}_{t, s}$, it is sufficient to define $\left.\ell\right|_{[0, t]}$, i.e. its values on $[0, t]$. The rest is given by (5.2) or (5.3). Defining $\ell$ on the whole interval $[0, T]$ instead of only on $[0, t]$ is only useful for notational simplicity.

Lemma 5.2 The sequence $\left(u^{n}\right)_{n}$ is non-increasing in $n$. Moreover, for bounded $u$, we have $\left\|u^{n}\right\|_{\infty} \leq$ $\|u\|_{\infty}$ for all $n \geq 0$. 
Proof The non-increase of the sequence $\left(u^{n}\right)_{n}$ is obvious. The inequality $u^{n} \leq\|u\|_{\infty}$ follows immediately from the definition of the regularization $u^{n}$. On the other hand, for $s>0$, we may find $\omega^{\varepsilon} \in \Omega$ such that $\left\|\eta_{s \wedge .}-\omega_{s \wedge}^{\varepsilon} .\right\|_{p+1} \rightarrow 0$ as $\varepsilon \rightarrow 0$. By taking $\theta=\left(s, \omega^{\varepsilon}\right)$ and $\ell=I$ (so that $\ell \in \mathcal{L}_{s, s}$ ) in (5.1), we get

$$
u^{n}(s, \eta) \geq \varlimsup_{\varepsilon \rightarrow 0}\left\{u\left(s, \omega^{\varepsilon}\right)-n\left\|\eta_{s \wedge \cdot}-\omega_{s \wedge}^{\varepsilon} \cdot\right\|_{p+1}^{p+1}\right\} \geq-\|u\|_{\infty} .
$$

In the remaining case $s=0$, we may find $\omega^{\varepsilon} \in \Omega$ such that $\left\|\eta_{0}-\omega_{\varepsilon \wedge}^{\varepsilon} \cdot\right\|_{p+1} \rightarrow 0$ as $\varepsilon \rightarrow 0$. Then, by taking $\theta=\left(\varepsilon, \omega^{\varepsilon}\right)$ and $\left.\ell\right|_{[0, \varepsilon]} \equiv 0$ (so that $\left.\ell \in \mathcal{L}_{\varepsilon, 0}\right)$ in (5.1), we also conclude that

$$
u^{n}(0, \eta) \geq \varlimsup_{\varepsilon \rightarrow 0}\left\{u\left(\varepsilon, \omega^{\varepsilon}\right)-n\left(\varepsilon^{\frac{2}{3 p+3}}+\left\|\eta_{0}-\omega_{\varepsilon \wedge}^{\varepsilon} \cdot\right\|_{p+1}^{p+1}\right)\right\} \geq-\|u\|_{\infty} .
$$

\subsection{Some properties of the regularization}

Our argument relies on using the regularization (5.1) for piecewise constant paths $\eta$ of the following form. Given $0=s_{1}<s_{2}<\cdots<s_{i} \leq T$ and $x_{1}, x_{2}, \cdots, x_{n} \in \mathbb{R}^{d}$, denote:

$$
\pi_{i}:=\left(s_{1}, \cdots, s_{i}\right), \quad \mathrm{x}_{i}:=\left(x_{1}, \cdots, x_{i}\right), \lambda_{i}:=\left(\pi_{i}, \mathrm{x}_{i-1}\right), \quad \text { and } \quad\left|\mathrm{x}_{i}\right|_{p}:=\left(\sum_{j=1}^{i}\left|x_{j}\right|^{p}\right)^{\frac{1}{p}},
$$

and define the corresponding piecewise constant path:

$$
\eta_{s}^{\lambda_{i}}(x)=\sum_{j=1}^{i-1} x_{j} 1_{\left\{s \geq s_{j}\right\}}+x 1_{\left\{s \geq s_{i}\right\}},
$$

i.e. $\eta^{\lambda_{i}}(x)$ is a càdlàg piecewise constant path with $j$-th jump of size $x_{j}$ at time $s_{j}$, for $j \leq i-1$, and a last jump of size $x$ at time $s_{i}$.

The following lemma provides an estimate on 1-optimal $(\hat{\theta}, \hat{\ell})$ in the definition of $u^{n}(s, \eta)$, in the case that $\eta=\eta^{\lambda_{i}}(x)$. For the sake of clarity, we recall the corresponding notion.

Definition 5.3 We say that $\left(\theta^{\delta}, \ell^{\delta}\right)$ is $\delta$-optimal in the definition of $u^{n}(s, \eta)$, if

$$
\ell^{\delta} \in \mathcal{L}_{t^{\delta}, s} \quad \text { and } \quad u^{n}(s, \eta)-u\left(\theta^{\delta}\right)+n \Phi\left(s, \eta, \theta^{\delta}, \ell^{\delta}\right)<\delta .
$$

We shall denote by 1 the column vector of ones with appropriate dimension, so that with the notations of (5.5), the flat tail of the path $\eta^{\lambda_{i}}\left(x_{i}\right)$ is given by:

$$
\eta_{s}^{\lambda_{i}}\left(x_{i}\right)=\mathrm{x}_{i} \mathbf{1}=x_{1}+\ldots+x_{i}, \quad s \geq s_{i}, \text { for all } i .
$$

Lemma 5.4 Let $\lambda_{i}$ be as in (5.5), $x \in \mathbb{R}^{d}, \mathrm{x}_{i}:=\left(\mathrm{x}_{\mathrm{i}-1}, x\right)$ (we slightly abuse the notation $\mathrm{x}_{i}$ so as to simplify the formulas below), and $s \in\left[s_{i}, T\right]$. Let $u$ be a bounded function. Then, for a 1-optimal point $(\hat{\theta}, \hat{\ell})$ for $u^{n}\left(s, \eta^{\lambda_{i}}(x)\right)$, we have:

$$
\left|\mathrm{x}_{i} \mathbf{1}-\hat{\omega}_{\hat{t}}\right| \leq C n^{-\frac{1}{p+1}},\|\hat{\ell}-I\|_{\infty} \leq C n^{-\frac{3 p+3}{2}} \text {, and }\left\|\eta^{\lambda_{i}}(x)-\hat{\omega}_{\hat{t} \wedge} \cdot\right\|_{p} \leq C\left(n^{-\frac{1}{p+1}}+i n^{-\frac{3 p+3}{2 p}}\left|\mathrm{x}_{i}\right|_{p}\right),
$$

for some constant $C$ depending only on $T$ and $\|u\|_{\infty}$. 
Proof Set $\eta:=\eta^{\lambda_{i}}(x)$. By the uniform bound on $u$ and $u^{n}$ in Lemma [5.2 it follows from (2.3) and (5.7) that

$$
\|\hat{\ell}-I\|_{\infty}^{\frac{2}{3 p+3}} \vee\left|\eta_{s}-\hat{\omega}_{\hat{t}}\right|^{p+1} \vee \int_{0}^{\hat{t}}\left|\eta_{\hat{\ell}(t)}-\hat{\omega}_{t}\right|^{p+1} d t \leq \frac{C_{0}}{n}:=\frac{1+2|u|_{\infty}}{n} .
$$

Since $\eta_{s}=\mathrm{x}_{i} \mathbf{1}$, this provides the first two required estimates.

By the Minkowski inequality, the Hölder inequality, and (2.3), we have

$$
\begin{aligned}
\|\eta-\hat{\omega} \cdot \wedge \hat{t}\|_{p} & \leq\left\|\eta-\eta_{\hat{\ell}(\cdot \hat{t})}\right\|_{p}+\left\|\eta_{\hat{\ell}(\wedge \hat{t})}-\hat{\omega}_{\cdot \wedge \hat{t}}\right\|_{p} \\
& \leq\left\|\eta-\eta_{\hat{\ell}(\wedge \hat{t})}\right\|_{p}+T^{\frac{1}{p(p+1)}}\left\|\eta_{\hat{\ell}(\cdot \wedge \hat{t})}-\hat{\omega}_{\cdot \wedge \hat{t}}\right\|_{p+1} \\
& =\left\|\eta-\eta_{\hat{\ell}(\cdot \wedge \hat{t})}\right\|_{p}+T^{\frac{1}{p(p+1)}}\left(\int_{0}^{\hat{t}}\left|\eta_{\hat{\ell}(t)}-\hat{\omega}_{t}\right|^{p+1} d t+(T-\hat{t})\left|\eta_{s}-\hat{\omega}_{\hat{t}}\right|^{p+1}\right)^{\frac{1}{p+1}} \\
& \leq\left\|\eta-\eta_{\hat{\ell}(\cdot \wedge \hat{t})}\right\|_{p}+\left((1+T)^{2} \frac{C_{0}}{n}\right)^{\frac{1}{p+1}}
\end{aligned}
$$

where the last inequality follows from (5.9). In the special case $s=s_{i}=0$, we have $\eta \equiv \eta_{0}$ and $\left.\hat{\ell}\right|_{[0, \hat{t}]} \equiv 0$, and we therefore get $\left\|\eta-\eta_{\hat{\ell}(. \wedge \hat{t})}\right\|_{p}=0$. Otherwise, in the case $s>0$, denoting $x_{i}:=x$, we have

$$
\begin{aligned}
\left\|\eta-\eta_{\hat{\ell}(\wedge \hat{t})}\right\|_{p}^{p} & =\int_{0}^{T}\left|\sum_{j=1}^{i} x_{j}\left(1_{\left\{t \geq s_{j}\right\}}-1_{\left\{\hat{\ell}(t \wedge \hat{t}) \geq s_{j}\right\}}\right)\right|^{p} d t \\
& \leq i^{p}\left(\sum_{j=1}^{i}\left|x_{j}\right|^{p} \int_{0}^{T}\left|1_{\left\{t \geq s_{j}\right\}}-1_{\left\{\hat{\ell}(t \wedge \hat{t}) \geq s_{j}\right\}}\right| d t\right) \\
& \leq i^{p}\left(\sum_{j=1}^{i}\left|x_{j}\right|^{p}\left|s_{j}-\hat{\ell}^{-1}\left(s_{j}\right)\right|\right) \leq i^{p}\left|\mathrm{x}_{i}\right|_{p}^{p}\|\hat{\ell}-I\|_{\infty} \leq i^{p}\left|\mathrm{x}_{i}\right|_{p}^{p}\left(\frac{C_{0}}{n}\right)^{\frac{3 p+3}{2}}
\end{aligned}
$$

by using again (5.9) . The third required estimate is obtained by plugging the last inequality into (5.10).

The previous lemma leads to:

Lemma 5.5 Let $u$ be bounded and $d_{p}$-uniformly continuous. Then, $\lim _{n \rightarrow \infty} u^{n}(\mathbf{0})=u(\mathbf{0})$.

Proof First, we clearly have

$$
u^{n}(\mathbf{0}) \geq \varlimsup_{\varepsilon \rightarrow 0}(u(\varepsilon, 0)-n \varepsilon)=u(\mathbf{0}) .
$$

On the other hand, for $\varepsilon<1$, choose an $\varepsilon$-optimal $(\hat{\theta}, \hat{\ell})$ in the definition of $u^{n}(0,0)$. It follows from Lemma 5.4 that

$$
d_{p}(\mathbf{0}, \hat{\theta})=|\hat{t}|+\left\|\hat{\omega}_{\hat{t} \wedge}\right\|_{p} \leq C\left(n^{-\frac{3 p+3}{2}}+n^{-\frac{1}{p+1}}\right)=: \delta_{n},
$$

where $C$ is a constant independent of $\varepsilon$, and thus $\delta_{n}$ does not depend on $\varepsilon$. Then

$$
\begin{aligned}
\left|\left(u^{n}-u\right)(\mathbf{0})\right| & =\left(u^{n}-u\right)(\mathbf{0}) \leq \varepsilon+u(\hat{\theta})-u(\mathbf{0}) \\
& \leq \varepsilon+\sup _{d_{p}(\theta, \mathbf{0}) \leq \delta_{n}}(u(\theta)-u(\mathbf{0})) \leq \varepsilon+\rho^{u}\left(\delta_{n}\right) \longrightarrow \rho^{u}\left(\delta_{n}\right),
\end{aligned}
$$

by the uniform continuity of $u$. Since $\delta_{n} \longrightarrow 0$, this shows that $\lim _{n \rightarrow \infty} u^{n}(\mathbf{0})=u(\mathbf{0})$. 


\subsection{A finite-dimensional regularization}

For any $\lambda_{i}$ as in (5.5), we now introduce the finite-dimensional function

$$
u^{n, \lambda_{i}}(s, x):=u^{n}\left(s, \eta^{\lambda_{i}}(x)\right), \quad(s, x) \in\left[s_{i}, T\right] \times \mathbb{R}^{d} .
$$

In this subsection, we explore the regularity of this function for fixed $\lambda_{i}$.

Notice that, for any $s, \lambda_{i}, x$, there exists a sequence $\left(\omega^{\varepsilon}\right)_{\varepsilon} \subset \Omega$ such that $\left\|\eta_{s \wedge}^{\lambda_{i}} .(x)-\omega_{s \wedge}^{\varepsilon} .\right\|_{p} \rightarrow 0$. Then, since $u, v, G$ are assumed to be uniformly continuous on $\Theta$, these functions have natural extensions for such càd-làg paths. Similar to (5.11), we denote

$$
u^{\lambda_{i}}(s, x):=u\left(s, \eta^{\lambda_{i}}(x)\right), \quad(s, x) \in\left[s_{i}, T\right] \times \mathbb{R}^{d} .
$$

Our first result provides an estimate of the deviation of the penalization at the final time $T$.

Lemma 5.6 Let $u$ be bounded and $d_{p}$-uniformly continuous. Then, for $\lambda_{i}$ as in (5.5), $x \in \mathbb{R}^{d}$, and $\mathrm{x}_{i}:=\left(\mathrm{x}_{\mathrm{i}-1}, x\right)$, we have

$$
\rho_{n}:=\sup \left\{\left|\left(u^{n, \lambda_{i}}-u^{\lambda_{i}}\right)(T, x)\right|: i \leq n^{1+\frac{1}{5 p}},\left|\mathrm{x}_{i}\right|_{p} \leq n^{\frac{1}{2}+\frac{6}{5 p}}\right\} \quad \longrightarrow \quad 0 \text { as } n \rightarrow \infty .
$$

Proof Set $\eta:=\eta^{\lambda_{i}}(x)$, and choose a sequence of $\left(\omega^{\varepsilon}\right)_{\varepsilon} \subset \Omega$ such that $\int_{0}^{T+1}\left|\eta_{t}-\omega_{t}^{\varepsilon}\right|^{p+1} d t \longrightarrow 0$, as $\varepsilon \rightarrow 0$. Then, we clearly have

$$
u^{n, \lambda_{i}}(T, x):=u^{n}(T, \eta) \geq \varlimsup_{\varepsilon \rightarrow 0} u\left(T, \omega^{\varepsilon}\right)=u^{\lambda_{i}}(T, x),
$$

where the last equality follows from the $d_{p}$-continuity of $u$.

On the other hand, choosing an $\varepsilon$-optimal $(\hat{\theta}, \hat{\ell})$ in the definition of $u^{n}(T, \eta)$, it follows from Lemma 5.4 that

$$
\begin{aligned}
d_{p}((T, \eta), \hat{\theta}) & =|T-\hat{t}|+\left\|\eta-\hat{\omega}_{\hat{t} \wedge} \cdot\right\|_{p} \\
& \leq C\left(n^{-\frac{3 p+3}{2}}+n^{-\frac{1}{p+1}}+i\left|\mathrm{x}_{i}\right|_{p} n^{-\frac{3 p+3}{2 p}}\right) \\
& \leq C\left(n^{-\frac{3 p+3}{2}}+n^{-\frac{1}{p+1}}+n^{1+\frac{1}{5 p}} n^{\frac{1}{2}+\frac{6}{5 p}} n^{-\frac{3 p+3}{2 p}}\right) \\
& \leq C\left(n^{-\frac{3 p+3}{2}}+n^{-\frac{1}{p+1}}+n^{-\frac{1}{10 p}}\right)=: \delta_{n}^{\prime} .
\end{aligned}
$$

For the second inequality, we used the constraints $i \leq n^{1+\frac{1}{5 p}}$, and $\left|\mathrm{x}_{i}\right|_{p} \leq n^{\frac{1}{2}+\frac{6}{5 p}}$. Then,

$$
\begin{aligned}
\left(u^{n, \lambda_{i}}-u^{\lambda_{i}}\right)(T, x) & \leq \varepsilon+u(\hat{\theta})-u(T, \eta) \\
& \leq \varepsilon+\sup _{d_{p}((T, \eta), \theta) \leq \delta_{n}^{\prime}}(u(\theta)-u(T, \eta)) \\
& \leq \varepsilon+\rho^{u}\left(\delta_{n}^{\prime}\right) \longrightarrow \rho^{u}\left(\delta_{n}^{\prime}\right), \text { as } \varepsilon \rightarrow 0 .
\end{aligned}
$$

Hence, we have $0 \leq\left(u^{n, \lambda_{i}}-u^{\lambda_{i}}\right)(T, x) \leq \rho^{u}\left(\delta_{n}^{\prime}\right)$, and the required result follows from the fact that $\delta_{n}^{\prime} \longrightarrow 0$ as $n \rightarrow \infty$.

We next analyze the regularity of the finite-dimensional regularization. 
Lemma 5.7 The function $u^{n, \lambda_{i}}$ is:

(i) $\frac{2}{3 p+3}-$ Hölder continuous in $s \in\left(s_{i}, T\right]$, uniformly in $x \in \mathbb{R}^{d}$,

(ii) locally Lipschitz-continuous in $x \in \mathbb{R}^{d}$, uniformly in $s \in\left(s_{i}, T\right]$,

(ii) lower semicontinuous at the points $\left(s_{i}, x\right), x \in \mathbb{R}^{d}$, i.e. $u^{n, \lambda_{i}}\left(s_{i}, x\right) \leq \underline{\lim }_{s^{\prime} \downarrow s_{i}, x^{\prime} \rightarrow x} u^{n, \lambda_{i}}\left(s^{\prime}, x^{\prime}\right)$.

Proof 1. We first consider $s, s^{\prime} \in\left(s_{i}, T\right]$, and we estimate the value of $u^{n, \lambda_{i}}(s, x)-u^{n, \lambda_{i}}\left(s^{\prime}, x^{\prime}\right)$ for $x, x^{\prime} \in \mathbb{R}^{d}$. Choose an $\varepsilon$-optimal $(\hat{\theta}, \hat{\ell})$ in the definition of $u^{n}\left(s, \eta^{\lambda_{i}}(x)\right)=u^{n, \lambda_{i}}(s, x)$. Given this $\hat{\ell} \in \mathcal{L}_{\hat{t}, s}$, we define $\hat{\ell}^{\prime} \in \mathcal{L}_{\hat{t}, s^{\prime}}$ by its values on $[0, \hat{t}]$ (see Remark [5.1):

$$
\hat{\ell}^{\prime}(t):=\hat{\ell}(t) \mathbf{1}_{\left[0, \hat{\ell}^{-1}\left(s_{i}\right)\right]}(t)+\left(s_{i}+\frac{s^{\prime}-s_{i}}{s-s_{i}}\left(\hat{\ell}(t)-s_{i}\right)\right) \mathbf{1}_{\left(\hat{\ell}^{-1}\left(s_{i}\right), t^{*}\right]}(t), \quad t \in[0, \hat{t}] .
$$

In particular, we observe that

$$
\left(\hat{\ell}^{\prime}\right)^{-1}\left(s_{i}\right)=\hat{\ell}^{-1}\left(s_{i}\right)
$$

and

$$
\begin{aligned}
\sup _{t \leq \hat{t}}\left|\hat{\ell}_{t}-\hat{\ell}_{t}^{\prime}\right| & \leq \sup _{\hat{\ell}^{-1}\left(s_{i}\right) \leq t \leq \hat{t}}\left|s_{i}+\left(\hat{\ell}(t)-s_{i}\right) \frac{s^{\prime}-s_{i}}{s-s_{i}}-\hat{\ell}(t)\right| \\
& \leq \sup _{\hat{\ell}^{-1}\left(s_{i}\right) \leq t \leq \hat{t}}\left(\hat{\ell}(t)-s_{i}\right) \frac{\left|s-s^{\prime}\right|}{s-s_{i}} \leq\left|s-s^{\prime}\right| .
\end{aligned}
$$

Then we have

$$
u^{n, \lambda_{i}}(s, x)-u^{n, \lambda_{i}}\left(s^{\prime}, x^{\prime}\right) \leq \varepsilon+n\left(\Phi\left(s^{\prime}, \eta^{\lambda}\left(x^{\prime}\right), \hat{\theta}, \hat{\ell}^{\prime}\right)-\Phi\left(s, \eta^{\lambda_{i}}(x), \hat{\theta}, \hat{\ell}\right)\right) .
$$

It follows from (5.13) that

$$
\left\|\hat{\ell}^{\prime}-I\right\|_{\infty}^{\frac{2}{3 p+3}}-\|\hat{\ell}-I\|_{\infty}^{\frac{2}{3 p+3}} \leq \sup _{t \leq \hat{t}}\left|\hat{\ell}_{t}-\hat{\ell}_{t}^{\prime}\right|^{\frac{2}{3 p+3}} \leq\left|s-s^{\prime}\right|^{\frac{2}{3 p+3}}
$$

Moreover, using (5.12), we directly estimate that:

$$
\begin{aligned}
& \left\|\eta^{\lambda_{i}}\left(x^{\prime}\right)_{\hat{\ell}(\hat{t} \wedge \cdot)}-\hat{\omega}_{\hat{t} \wedge \cdot}\right\|_{p+1}^{p+1}-\left\|\eta^{\lambda_{i}}(x)_{\hat{\ell}(\hat{t} \wedge \cdot)}-\hat{\omega}_{\hat{t} \wedge \cdot} \cdot\right\|_{p+1}^{p+1} \\
\leq & \int_{\hat{\ell}^{-1}\left(s_{i}\right)}^{T+1}\left|\sum_{j=1}^{i-1} x_{j}+x^{\prime}-\omega_{t \wedge t^{*}}^{*}\right|^{p+1} d t-\int_{\hat{\ell}^{-1}\left(s_{i}\right)}^{T+1}\left|\sum_{j=1}^{i-1} x_{j}+x-\omega_{t \wedge t^{*}}^{*}\right|^{p+1} d t \\
\leq & (p+1)\left|x-x^{\prime}\right| \int_{0}^{T+1}\left(\left|x-x^{\prime}\right|+\left|\sum_{j=1}^{i-1} x_{j}+x-\hat{\omega}_{t \wedge \hat{t}}\right|\right)^{p} d t .
\end{aligned}
$$

In view of the control on $\left\|\hat{\omega}_{\hat{t} \wedge} .\right\|_{p} \leq C$ from Lemma 5.4 this provides the statements (i) and (ii) by plugging (5.15) and (5.16) into (5.14).

2. We next estimate the difference of $u^{n, \lambda_{i}}\left(s_{i}, x\right)-u^{n, \lambda_{i}}\left(s^{\prime}, x^{\prime}\right)$ for $s^{\prime} \in\left(s_{i}, T\right]$. We consider two alternative cases.

Case 1: $i>1$. Then, $s_{i}>0$. Choose an $\varepsilon$-optimal $(\hat{\theta}, \hat{\ell})$ in the definition of $u^{n, \lambda_{i}}\left(s_{i}, x\right)$. Given this $\hat{\ell} \in \mathcal{L}_{\hat{t}, s_{i}}$, we define $\hat{\ell}^{\prime} \in \mathcal{L}_{\hat{t}+\varepsilon, s^{\prime}}$ by:

$$
\hat{\ell}^{\prime}(t):=\hat{\ell}(t) \quad \text { for } t \leq \hat{\ell}^{-1}\left(s_{i}\right)=\hat{t}, \quad \text { and } \quad \hat{\ell}^{\prime} \text { linear on }[\hat{t}, \hat{t}+\varepsilon] \text {. }
$$


In particular, we note that $\left(\hat{\ell}^{\prime}\right)^{-1}\left(s_{i}\right)=\hat{\ell}^{-1}\left(s_{i}\right)$. Then we have

$$
\begin{aligned}
u^{n, \lambda_{i}}\left(s_{i}, x\right)-u^{n, \lambda_{i}}\left(s^{\prime}, x^{\prime}\right) \leq & \varepsilon+u(\hat{\theta})-u\left(\hat{t}+\varepsilon, \hat{\omega}_{\hat{t} \wedge}\right) \\
& +n\left(\Phi\left(s^{\prime}, \eta^{\lambda_{i}}\left(x^{\prime}\right), \hat{t}+\varepsilon, \hat{\omega}_{\hat{t} \wedge .}, \ell^{\prime}\right)-\Phi\left(s_{i}, \eta^{\lambda_{i}}(x), \hat{\theta}, \hat{\ell}\right)\right) .
\end{aligned}
$$

Note that

$$
\begin{aligned}
\left\|\hat{\ell}^{\prime}-I\right\|_{\infty}^{\frac{2}{3 p+3}}-\|\hat{\ell}-I\|_{\infty}^{\frac{2}{3 p+3}} & \leq\left(\|\hat{\ell}-I\|_{\infty} \vee\left|s^{\prime}-\hat{t}-\varepsilon\right|\right)^{\frac{2}{3 p+3}}-\|\hat{\ell}-I\|_{\infty}^{\frac{2}{3 p+3}} \\
& \leq\left(\max \left\{0,\left|s^{\prime}-\hat{t}-\varepsilon\right|-\left\|\hat{\ell}-I_{\hat{t}}\right\|_{\infty}\right\}\right)^{\frac{2}{3 p+3}} \\
& \leq\left(\max \left\{0,\left|s^{\prime}-\hat{t}-\varepsilon\right|-\left|s_{i}-\hat{t}\right|\right\}\right)^{\frac{2}{3 p+3}} \\
& \leq\left(\left|s_{i}-s^{\prime}\right|+\varepsilon\right)^{\frac{2}{3 p+3}} .
\end{aligned}
$$

Further, by following the line of calculation in (5.16), we obtain that for all $|x|,\left|x^{\prime}\right| \leq R$ there is a constant $C$ (dependent on $R$, but independent of $n$ ) such that

$$
\begin{aligned}
u^{n, \lambda_{i}}\left(s_{i}, x\right)-u^{n, \lambda_{i}}\left(s^{\prime}, x^{\prime}\right) & \leq \varepsilon+\rho^{u}(\varepsilon)+n\left(\left(\left|s_{i}-s^{\prime}\right|+\varepsilon\right)^{\frac{2}{3 p+3}}+C\left|x-x^{\prime}\right|\right) \\
& \longrightarrow n\left(\left|s_{i}-s^{\prime}\right|^{\frac{2}{3 p+3}}+C\left|x-x^{\prime}\right|\right) .
\end{aligned}
$$

This implies that (iii) holds in the present.

Case 2: $i=1$. Then, $s_{i}=0$ and $\eta^{\lambda_{i}}(x) \equiv x$. Choose an $\varepsilon$-optimal $(\hat{\theta}, \hat{\ell})$ in the definition of $u^{n, \lambda_{i}}(0, x)$. Since $\hat{\ell} \in \mathcal{L}_{\hat{t}, 0}$, we have $\left.\hat{\ell}\right|_{[0, \hat{t}]} \equiv 0$. Assume $\varepsilon<s^{\prime}$, and define $\ell^{\prime} \in \mathcal{L}_{\hat{t}+\varepsilon, s^{\prime}}$ :

$$
\hat{\ell}^{\prime}(t):=\varepsilon_{\hat{\hat{t}}}^{t} \mathbf{1}_{[0, \hat{t}]}(t)+\left(\hat{t}+\varepsilon-t+s^{\prime} \frac{t-\hat{t}}{\varepsilon}\right) \mathbf{1}_{(\hat{t}, \hat{t}+\varepsilon]}(t), \quad t \in[0, \hat{t}+\varepsilon] .
$$

Then we have

$$
u^{n, \lambda_{i}}(0, x)-u^{n, \lambda_{i}}\left(s^{\prime}, x^{\prime}\right) \leq \varepsilon+u(\hat{\theta})-u\left(\hat{t}+\varepsilon, \hat{\omega}_{\hat{t} \wedge}\right)+n\left(\Phi\left(s^{\prime}, x^{\prime}, \hat{t}+\varepsilon, \hat{\omega}_{\hat{t} \wedge \cdot}, \hat{\ell}^{\prime}\right)-\Phi(0, x, \hat{\theta}, \hat{\ell})\right) .
$$

Note that

$$
\begin{aligned}
\left\|\hat{\ell}^{\prime}-I\right\|_{\infty}^{\frac{2}{3+3}}-\|\hat{\ell}-I\|_{\infty}^{\frac{2}{3 p+3}} & \leq\left(|\hat{t}-\varepsilon| \vee\left|s^{\prime}-\hat{t}-\varepsilon\right|\right)^{\frac{2}{3 p+3}}-|\hat{t}|^{\frac{2}{3 p+3}} \\
& \leq\left(\max \left\{\varepsilon,\left|s^{\prime}-\hat{t}-\varepsilon\right|-|\hat{t}|\right\}\right)^{\frac{2}{3 p+3}} \\
& \leq\left(\left|s^{\prime}\right|+\varepsilon\right)^{\frac{2}{3 p+3}}
\end{aligned}
$$

Then following the same line of calculation as in (5.16), we can again verify that (iii) also holds in this case.

\subsection{Viscosity solution property of the regularized solutions}

In this subsection, we prove a crucial property of the regularization (5.1). Namely, the induced finite-dimensional function $u^{n, \lambda_{i}}$ is a Crandall-Lions viscosity subsolution of the corresponding PDE 
with an appropriate error term. We recall that this does not imply the stronger claim that $u^{n, \lambda_{i}}$ is a $\mathcal{P}$ - viscosity subsolution, since the last notion involves a larger set of test functions. This is a major difference between the approach of this paper and the one followed in our previous paper [21] focused on semilinear path-dependent PDEs.

Proposition 5.8 Let $\lambda_{i}$ be as in (5.5), and $u$ be a $\mathcal{P}_{L}$-viscosity subsolution of PPDE (3.2) on $[0, T) \times \Omega$. Then $u^{n, \lambda_{i}}$ is a Crandall-Lions viscosity subsolution of the PDE:

$$
-\partial_{s} u^{n, \lambda_{i}}-G\left(s, \eta^{\lambda_{i}}(x), u^{n, \lambda_{i}}, D u^{n, \lambda_{i}}, D^{2} u^{n, \lambda_{i}}\right)-\alpha^{n}(s)-\beta^{u, n}(x) \leq 0, \quad \text { on } \quad\left(s_{i}, T\right) \times \mathbb{R}^{d} .
$$

where, for some constant $C>0, \alpha^{n}$ and $\beta^{n}$ are given by:

$$
\begin{gathered}
\alpha^{n}(s):=C\left(n\left|s-s_{i}\right|+\left(n\left|s-s_{i}\right|\right)^{\frac{1}{p+1}}+n^{-\frac{1}{2}}\right), \\
\beta^{u, n}(x):=\left(\rho^{G}+L_{0} \rho^{u}\right)\left(C\left(n^{-\frac{1}{p+1}}+i\left|\left(\mathrm{x}_{i-1}, x\right)\right|_{p} n^{-\frac{3 p+3}{2 p}}\right)\right) .
\end{gathered}
$$

Proof Let $(s, x) \in\left[s_{i}, T\right) \times \mathbb{R}^{d}$, and $(\alpha, \beta, \gamma) \in \underline{J} u^{n, \lambda_{i}}(s, x)$. Then for all $\varepsilon>0$,

$$
u^{n, \lambda_{i}}(s, x)=\max _{t \in[s, s+h],|y-x| \leq h}\left\{u^{n, \lambda_{i}}(t, y)-(\alpha+\varepsilon)(t-s)-\beta \cdot(y-x)-\frac{1}{2}(y-x)^{\mathrm{T}}\left(\gamma+\varepsilon \mathbb{I}_{d}\right)(y-x)\right\},
$$

for some $h \in(0,1)$, where $\mathbb{I}_{d}$ is the $d \times d$-identity matrix. Let $\mathrm{H}:=\mathrm{H}_{\delta} \leq h$ be a stopping time as in (3.3), for some $\delta<h$ to be chosen later. From (5.19), we deduce that

$$
u^{n, \lambda_{i}}(s, x)>\mathrm{E}:=\overline{\mathcal{E}}_{L}\left[u^{n, \lambda_{i}}\left(s+\mathrm{H}, x+B_{\mathrm{H}}\right)-(\alpha+2 \varepsilon) \mathrm{H}-\beta \cdot B_{\mathrm{H}}-\frac{1}{2} B_{\mathrm{H}}^{\mathrm{T}}\left(\gamma+\varepsilon \mathbb{I}_{d}\right) B_{\mathrm{H}}\right] .
$$

Our objective is to deduce from this inequality an appropriate point in the $\mathcal{P}$-subjet of $u$.

(i) For $\hat{\varepsilon}:=u^{n, \lambda_{i}}(s, x)-\mathrm{E}$, let $(\tilde{\theta}, \tilde{\ell})$ be a $(1 \wedge \hat{\varepsilon})$-maximizer in the definition of $u^{n, \lambda_{i}}(s, x)$. Then

$$
\mathrm{E}<u(\tilde{\theta})-n \Phi\left(s, \eta^{\lambda_{i}}(x), \tilde{\theta}, \tilde{\ell}\right) .
$$

We recall from Lemma 5.4 that, with $\mathrm{x}_{i}:=\left(\mathrm{x}_{i-1}, x\right)$, we have

$$
\left|\mathrm{x}_{i} \mathbf{1}-\tilde{\omega}_{\tilde{t}}\right| \leq C n^{-\frac{1}{p+1}} \quad \text { and } \quad d_{p}\left(\left(s, \eta^{\lambda_{i}}(x)\right), \tilde{\theta}\right) \leq C\left(n^{-\frac{1}{p+1}}+i n^{-\frac{3 p+3}{2 p}}\left|\mathrm{x}_{i}\right|_{p}\right) .
$$

On the other hand, it follows from the definition of $u^{n}$ in (5.1) that

$$
u^{n, \lambda_{i}}\left(s+\mathrm{H}, x+B_{\mathrm{H}}\right) \geq u^{\tilde{\theta}}(\mathrm{H}, B)-n \Phi\left(s+\mathrm{H}, \eta^{\lambda_{i}}(x+\mathrm{H}), \tilde{\theta}, \tilde{\ell}\right) .
$$

Combining (5.20), (5.21) and (5.23), we get

$$
u(\tilde{\theta})>\overline{\mathcal{E}}_{L}\left[u^{\tilde{\theta}}(\mathrm{H}, B)-(\alpha+2 \varepsilon) \mathrm{H}-\beta \cdot B_{\mathrm{H}}-\frac{1}{2} B_{\mathrm{H}}^{\mathrm{T}}\left(\gamma+\varepsilon \mathbb{I}_{d}\right) B_{\mathrm{H}}+n \Delta \Phi\right],
$$

where

$$
\begin{aligned}
\Delta \Phi & :=\Phi\left(s, \eta^{\lambda_{i}}(x), \tilde{\theta}, \tilde{\ell}\right)-\Phi\left(s+\mathrm{H}, \eta^{\lambda_{i}}(x+\mathrm{H}), \tilde{\theta}, \tilde{\ell}\right) \\
& =\int_{0}^{T+1}\left|\eta^{\lambda_{i}}(x)_{s \wedge \tilde{\ell}(t)}-\tilde{\omega}_{\tilde{t} \wedge t}\right|^{p+1} d t-\int_{0}^{T+1}\left|\eta^{\lambda_{i}}\left(x+B_{\mathrm{H}}\right)_{s \wedge \tilde{\ell}(t)}-\tilde{\omega}_{\tilde{t} \wedge t}\right|^{p+1} d t .
\end{aligned}
$$


(ii) In this step, we derive an appropriate minorant of $\Delta \Phi$. Since $\tilde{\ell} \in \mathcal{L}_{\tilde{t}, s}$, this difference reduces to

$$
\begin{aligned}
\Delta \Phi= & \int_{\tilde{\ell}^{-1}\left(s_{i}\right)}^{T+1}\left|\eta^{\lambda_{i}}(x)_{s \wedge \tilde{\ell}(t)}-\tilde{\omega}_{\tilde{t} \wedge t}\right|^{p+1} d t-\int_{\tilde{\ell}^{-1}\left(s_{i}\right)}^{T+1}\left|\eta^{\lambda_{i}}\left(x+B_{\mathrm{H}}\right)_{s \wedge \tilde{\ell}(t)}-\tilde{\omega}_{\tilde{t} \wedge t}\right|^{p+1} d t \\
= & \int_{\tilde{\ell}^{-1}\left(s_{i}\right)}^{\tilde{t}}\left(\left|\bar{x}-\tilde{\omega}_{t}\right|^{p+1}-\left|\mathrm{x}_{i} \mathbf{1}+B_{\mathrm{H}}-\tilde{\omega}_{t}\right|^{p+1}\right) d t \\
& \quad+\int_{\tilde{t}}^{\tilde{t}+\mathrm{H}}\left(\left|\mathrm{x}_{i} \mathbf{1}-\tilde{\omega}_{\tilde{t}}\right|^{p+1}-\left|\mathrm{x}_{i} \mathbf{1}+B_{\mathrm{H}}-\tilde{\omega}_{\tilde{t}}-B_{t-\tilde{t}}\right|^{p+1}\right) d t .
\end{aligned}
$$

We next use the obvious identity $\left|a^{p+1}-b^{p+1}\right| \leq|a-b| \sum_{j=0}^{p}|a|^{j}|b|^{p-j} \leq(p+1)|a-b|(|a|+|b|)^{p}$. This together with (5.22) allows to control the integrand of the second term:

$$
\begin{aligned}
|| \mathrm{x}_{i} \mathbf{1}-\left.\tilde{\omega}_{\tilde{t}}\right|^{p+1}-\left|\mathrm{x}_{i} \mathbf{1}+B_{\mathrm{H}}-\tilde{\omega}_{\tilde{t}}-B_{t-\tilde{t}}\right|^{p+1} \mid & \leq(p+1)\left|B_{\mathrm{H}}-B_{t-\tilde{t}}\right|\left(2\left|\mathrm{x}_{i} \mathbf{1}-\tilde{\omega}_{\tilde{t}}\right|+\left|B_{\mathrm{H}}-B_{t-\tilde{t}}\right|\right)^{p} \\
& \leq(p+1)\left|B_{\mathrm{H}}-B_{t-\tilde{t}}\right|\left(2 C n^{-\frac{1}{p+1}}+\left|B_{\mathrm{H}}-B_{t-\tilde{t}}\right|\right)^{p} .
\end{aligned}
$$

Since $0 \leq t-\tilde{t} \leq \mathrm{H}=\mathrm{H}_{\delta}$, we see that $\left|B_{\mathrm{H}}-B_{t-\tilde{t}}\right| \leq 2 \delta$. We then obtain for sufficiently small $\delta$ :

$$
|| \mathrm{x}_{i} \mathbf{1}-\left.\tilde{\omega}_{\tilde{t}}\right|^{p+1}-\left|\mathrm{x}_{i} \mathbf{1}+B_{\mathrm{H}}-\tilde{\omega}_{\tilde{t}}-B_{t-\tilde{t}}\right|^{p+1} \mid \leq 2 \delta(p+1)\left(2 C n^{-\frac{1}{p+1}}+2 \delta\right)^{p}<\varepsilon n^{-1} .
$$

Therefore,

$$
\begin{aligned}
\Delta \Phi & \geq-\varepsilon n^{-1} \mathrm{H}+\int_{\tilde{\ell}^{-1}\left(s_{i}\right)}^{\tilde{t}}\left(\left|\mathrm{x}_{i} \mathbf{1}-\tilde{\omega}_{t}\right|^{p+1}-\left|\mathrm{x}_{i} \mathbf{1}+B_{\mathrm{H}}-\tilde{\omega}_{t}\right|^{p+1}\right) d t \\
& \underset{(\star)}{\geq}-\varepsilon n^{-1} \mathrm{H}-\int_{\tilde{\ell}^{-1}\left(s_{i}\right)}^{\tilde{t}}\left[(p+1) B_{\mathrm{H}} \cdot\left(\mathrm{x}_{i} \mathbf{1}-\tilde{\omega}_{t}\right)\left|\mathrm{x}_{i} \mathbf{1}-\tilde{\omega}_{t}\right|^{p-1}+C\left|B_{\mathrm{H}}\right|^{2}\left(\left|B_{\mathrm{H}}\right|^{p-1}+\left|\mathrm{x}_{i} \mathbf{1}-\tilde{\omega}_{t}\right|^{p-1}\right)\right] d t
\end{aligned}
$$

where the last inequality $(\star)$ follows from an easy calculation reported in Step (v) below. Since $\left|B_{\mathrm{H}}\right| \leq \delta \leq 1$, this provides

$$
\begin{aligned}
\Delta \Phi & \geq-\varepsilon n^{-1} \mathrm{H}-\int_{\tilde{\ell}^{-1}\left(s_{i}\right)}^{\tilde{t}}\left[(p+1) B_{H} \cdot\left(\mathrm{x}_{i} \mathbf{1}-\tilde{\omega}_{t}\right)\left|\mathrm{x}_{i} \mathbf{1}-\tilde{\omega}_{t}\right|^{p-1}+C\left|B_{H}\right|^{2}\left(1+\left|\mathrm{x}_{i} \mathbf{1}-\tilde{\omega}_{t}\right|^{p-1}\right)\right] d t \\
& \geq-\varepsilon n^{-1} \mathrm{H}-\int_{\tilde{\ell}^{-1}\left(s_{i}\right)}^{\tilde{t}}\left[(p+1) B_{H} \cdot\left(\mathrm{x}_{i} \mathbf{1}-\tilde{\omega}_{t}\right)\left|\mathrm{x}_{i} \mathbf{1}-\tilde{\omega}_{t}\right|^{p-1}+C\left|B_{H}\right|^{2}\left(2+\left|\mathrm{x}_{i} \mathbf{1}-\tilde{\omega}_{t}\right|^{p}\right)\right] d t .
\end{aligned}
$$

(iii) We now deduce from the previous step the corresponding minorant of $\mathbb{E}^{\mathbb{P}}[\Delta \Phi]$, for an arbitrary $\mathbb{P} \in \mathcal{P}_{L}$. By Lemma 2.1, we deduce from (5.25) that

$$
\mathbb{E}^{\mathbb{P}}[\Delta \Phi] \geq-\mathbb{E}^{\mathbb{P}}[\mathrm{H}]\left(\varepsilon n^{-1}+C \int_{\tilde{\ell}^{-1}\left(s_{i}\right)}^{\tilde{t}}\left|\mathrm{x}_{i} \mathbf{1}-\tilde{\omega}_{t}\right|^{p} d t+C\left|\tilde{t}-\tilde{\ell}^{-1}\left(s_{i}\right)\right|\right) .
$$

We shall verify in Steps (vi) and (vii) below that the following estimates hold:

$$
\begin{gathered}
\left|\tilde{t}-\tilde{\ell}^{-1}\left(s_{i}\right)\right| \leq C n^{-\frac{3 p+3}{2}}+\left|s-s_{i}\right|, \\
\int_{\tilde{\ell}^{-1}\left(s_{i}\right)}^{\tilde{t}}\left|\mathbf{x}_{i} \mathbf{1}-\tilde{\omega}_{t}\right|^{p} d t \leq C n^{-\frac{p}{p+1}}\left(n^{-\frac{3 p+3}{2}}+\left|s-s_{i}\right|\right)^{\frac{1}{p+1}},
\end{gathered}
$$


where $C$ is a positive constant independent of $n$. Then

$$
n \mathbb{E}^{\mathbb{P}}[\Delta \Phi] \geq-\mathbb{E}^{\mathbb{P}}[\mathrm{H}]\left(\varepsilon+\alpha^{n}(s)\right),
$$

where $\alpha^{n}(s)$ is as defined in (5.17).

(iv) We are now ready to prove the required result. Plugging the last minorant in (5.24), we see that

$$
u(\tilde{\theta})>\overline{\mathcal{E}}_{L}\left[u^{\tilde{\theta}}(\mathrm{H}, B)-\left(\alpha+\alpha^{n}(s)+3 \varepsilon\right) \mathrm{H}-\beta \cdot B_{\mathrm{H}}-\frac{1}{2} B_{\mathrm{H}}^{\mathrm{T}}\left(\gamma+\varepsilon \mathbb{I}_{d}\right) B_{\mathrm{H}}\right] .
$$

By Lemma 3.3 , there is a point $\theta^{*}$ such that

$$
t^{*}<\mathrm{H}\left(\omega^{*}\right) \text { and }\left(\alpha+\alpha^{n}(s)+3 \varepsilon, \beta+\left(\gamma+\varepsilon \mathbb{I}_{d}\right) \omega_{t^{*}}^{*}, \gamma+\varepsilon \mathbb{I}_{d}\right) \in \underline{\mathcal{J}}_{L} u\left(\tilde{t}+t^{*}, \tilde{\omega} \otimes_{\tilde{t}} \omega^{*}\right) .
$$

By choosing $\delta$ small enough, we have

$$
\begin{gathered}
\left|\left(\gamma+\varepsilon \mathbb{I}_{d}\right) \omega_{t^{*}}^{*}\right| \leq\left|\gamma+\varepsilon \mathbb{I}_{d}\right| \delta \leq \frac{\varepsilon}{L_{0}} \\
d_{p}\left(\tilde{\theta},\left(\tilde{t}+t^{*}, \tilde{\omega} \otimes_{\tilde{t}} \omega^{*}\right)\right)=t^{*}+\left(\int_{\tilde{t}}^{T+1} \mid \omega_{(t-\tilde{t}) \wedge t^{*}}^{*} d t\right)^{\frac{1}{p}} \leq C \delta \leq \varepsilon .
\end{gathered}
$$

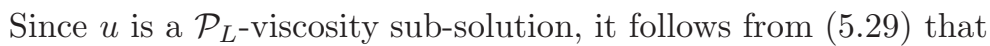

$$
-\alpha-\alpha^{n}(s)-3 \varepsilon-G\left(\tilde{t}+t^{*}, \tilde{\omega} \otimes_{\tilde{t}} \omega^{*}, u, \beta+\left(\gamma+\varepsilon \mathbb{I}_{d}\right) \omega_{t^{*}}^{*}, \gamma+\varepsilon \mathbb{I}_{d}\right) \leq 0 .
$$

Recall that $u$ is $d_{p}$-uniformly continuous. By using Assumption 4.1 and the estimates (5.22), (5.30) and (5.31), we deduce from the last inequality that

$$
-\alpha-\alpha^{n}(s)-4 \varepsilon-\beta^{u, n}(x)-G\left(s, \eta^{\lambda_{i}}(x), u\left(s, \eta^{\lambda_{i}}(x)\right), \beta, \gamma+\varepsilon \mathbb{I}_{d}\right) \leq 0,
$$

where $\beta^{u, n}(x)$ is as defined in (5.18). Finally, sending $\varepsilon \rightarrow 0$ and using the monotonicity assumption in Remark 4.3, we obtain

$$
-\alpha-\alpha^{n}(s)-\beta^{u, n}(x)-G\left(s, \eta^{\lambda_{i}}(x), u^{n, \lambda_{i}}(x), \beta, \gamma\right) \leq 0 .
$$

(v) Proof of $(\star)$ Clearly, this inequality is implied by

$|a+b|^{p+1} \leq|a|^{p+1}+(p+1) a \cdot b|a|^{p-1}+C|b|^{2}\left(|b|^{p-1}+|a|^{p-1}\right), \quad$ for $a, b \in \mathbb{R}^{d}, \quad$ for some $C \geq 0$, which we now verify. Since $p>1$ is odd, see Remark 4.4 we have

$$
|a+b|^{p+1}=|a|^{p+1}+(p+1) a \cdot b|a|^{p-1}+R, \quad \text { where } \quad R:=\sum_{k+j \leq \frac{p+1}{2}, k+2 j \geq 2}(a \cdot b)^{k}|b|^{2 j}|a|^{p+1-2 k-2 j} .
$$

The required inequality follows from the existence of a constant $C$, depending only on $p$, such that

$$
|R| \leq C \sum_{k=2}^{p+1}|b|^{k}|a|^{p+1-k} \leq C|b|^{2} \sum_{k=0}^{p-1}|b|^{k}|a|^{p-1-k} \leq C p|b|^{2}\left(|b|^{p-1}+|a|^{p-1}\right) .
$$


(vi) Proof of (5.27). Recall the estimates in (5.8). Since $\tilde{\ell} \in \mathcal{L}_{\tilde{t}, s}$, we have

$$
\begin{aligned}
\left|\tilde{t}-\tilde{\ell}^{-1}\left(s_{i}\right)\right| & =\left|\tilde{\ell}^{-1}(s)-\tilde{\ell}^{-1}\left(s_{i}\right)\right| \\
& \leq\left|\tilde{\ell}^{-1}(s)-s\right|+\left|s-s_{i}\right|+\left|\tilde{\ell}^{-1}\left(s_{i}\right)-s_{i}\right| \\
& \leq C n^{-\frac{3 p+3}{2}}+\left|s-s_{i}\right| .
\end{aligned}
$$

For the last inequality, we used the fact that $(\tilde{\theta}, \tilde{\ell})$ is 1-optimal in the definition of $u^{n, \lambda_{i}}(s, x)$.

(vii) Proof of (5.28). We directly estimate that

$$
\begin{aligned}
\int_{\tilde{\ell}^{-1}\left(s_{i}\right)}^{\tilde{t}}\left|\mathrm{x}_{i} \mathbf{1}-\tilde{\omega}_{t}\right|^{p} d t & \leq\left(\int_{\tilde{\ell}^{-1}\left(s_{i}\right)}^{\tilde{t}}\left|\mathrm{x}_{i} \mathbf{1}-\tilde{\omega}_{t}\right|^{p+1} d t\right)^{\frac{p}{p+1}}\left(\tilde{t}-\tilde{\ell}^{-1}\left(s_{i}\right)\right)^{\frac{1}{p+1}} \\
& \leq C n^{-\frac{p}{p+1}}\left(n^{-\frac{3 p+3}{2}}+\left|s-s_{i}\right|\right)^{\frac{1}{p+1}}
\end{aligned}
$$

where the last inequality follows from (5.27) together with the 1-optimality of $(\tilde{\theta}, \tilde{\ell})$.

\section{Comparison result}

In this section, we fix $a, m:=m_{n} \in \mathbb{N}$, and the partition $\left(s_{i}^{n}\right)_{i}$ as follows:

$$
0<a<(5 p)^{-1}, \quad m_{n}:=\left\lfloor n^{1+a}+1\right\rfloor, \quad \text { and } \quad s_{i}^{n}:=(i-1) m_{n}^{-1} T, \quad i=1, \ldots, m_{n}+1,
$$

where $\lfloor\alpha\rfloor$ denotes the largest integer minorant of $\alpha$. We fix a piecewise constant path with jumps occurring at $\left\{s_{j}^{n}\right\}_{j \leq i}$, for all $i \leq m_{n}$ :

$$
\eta^{\lambda_{i}^{n}}(x) \quad \text { with } \quad \lambda_{i}^{n}:=\left(\pi_{i}^{n}, \mathrm{x}_{i-1}^{n}\right), \pi_{i}^{n}:=\left(s_{1}^{n}, \cdots, s_{i}^{n}\right), \text { and } \mathrm{x}_{i-1}^{n}:=\left(x_{1}^{n}, \cdots, x_{i-1}^{n}\right) \in \mathbb{R}^{i-1} .
$$

The following is a direct corollary of Proposition 5.8 .

Corollary 6.1 Function $u^{n, \lambda_{i}^{n}}$ is a Crandall-Lions viscosity subsolution of the PDE:

$$
-\partial_{s} u^{n, \lambda_{i}^{n}}-G\left(s, \eta^{\lambda_{i}^{n}}(x), u^{n, \lambda_{i}^{n}}, D u^{n, \lambda_{i}^{n}}, D^{2} u^{n, \lambda_{i}^{n}}\right)-R^{u, n}(x) \leq 0, \quad \text { on }\left(s_{i}^{n}, s_{i+1}^{n}\right) \times \mathbb{R}^{d},
$$

where $R^{u, n}(x):=C n^{-\frac{a}{p+1}}+\beta^{u, n}(x)$. Moreover

(i) $u^{n, \lambda_{i}^{n}}\left(s_{i+1}^{n}, x_{i+1}^{n}\right)=u^{n, \lambda_{i+1}^{n}}\left(s_{i+1}^{n}, 0\right)$,

(ii) $u^{n, \lambda_{i}^{n}}$ is locally $\frac{2}{3 p+3}-$ Hölder-continuous in $s$, Lipschitz-continuous in $x$ on $\left(s_{i}^{n}, s_{i+1}^{n}\right)$,

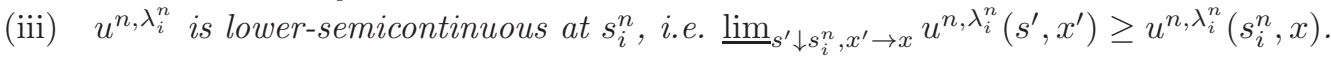

We next state the similar result for supersolutions. Let $v$ be a bounded and uniformly continuous $\mathcal{P}_{L}$-viscosity supersolution. Then we introduce the regularization:

$$
v^{n}(s, \eta):=\inf _{\theta \in \Theta \backslash \mathbf{0}, \ell \in \mathcal{L}_{t, s}}\{v(\theta)+n \Phi(s, \eta, \theta, \ell)\} .
$$

By the same arguments as in the previous section, we have that the function $v^{n, \lambda_{i}^{n}}$ satisfies the corresponding symmetric properties. 
Corollary 6.2 Function $v^{n, \lambda_{i}^{n}}$ is a Crandall-Lions viscosity supersolution of the PDE:

$$
-\partial_{s} v^{n, \lambda_{i}^{n}}-G\left(s, \eta^{\lambda_{i}^{n}}(x), v^{n, \lambda_{i}^{n}}, D v^{n, \lambda_{i}^{n}}, D^{2} v^{n, \lambda_{i}^{n}}\right)+R^{v, n}(x) \geq 0, \quad \text { on }\left(s_{i}^{n}, s_{i+1}^{n}\right) \times \mathbb{R}^{d},
$$

where $R^{v, n}(x):=C n^{-\frac{a}{p+1}}+\beta^{v, n}(x)$. Moreover

(i) $v^{n, \lambda_{i}^{n}}\left(s_{i+1}^{n}, x_{i+1}^{n}\right)=v^{n, \lambda_{i+1}^{n}}\left(s_{i+1}^{n}, 0\right)$,

(ii) $v^{n, \lambda_{i}^{n}}$ is locally $\frac{2}{3 p+3}-$ Hölder-continuous in s, Lipschitz-continuous in $x$ on $\left(s_{i}^{n}, s_{i+1}^{n}\right)$,

(iii) $v^{n, \lambda_{i}^{n}}$ is upper-semicontinuous at $s_{i}^{n}$, i.e. $\varlimsup_{\lim _{s^{\prime}} \downarrow s_{i}^{n}, x^{\prime} \rightarrow x} v^{n, \lambda_{i}^{n}}\left(s^{\prime}, x^{\prime}\right) \leq v^{n, \lambda_{i}^{n}}\left(s_{i}^{n}, x\right)$.

As a final ingredient for our proof of the comparison result, we introduce for $(s, x) \in\left[s_{i}^{n}, T\right] \times \mathbb{R}^{d}$ :

$$
\begin{aligned}
& u^{n, \lambda_{i}^{n}, \kappa}(s, x):=e^{2 L_{0} s} u^{n, \lambda_{i}^{n}}(s, x)-\frac{\kappa n^{-1-a}}{s-s_{i}^{n}}-\frac{1}{2 n}|x|^{2}, \\
& v^{n, \lambda_{i}^{n}, \kappa}(s, x):=e^{2 L_{0} s} v^{n, \lambda_{i}^{n}}(s, x)+\frac{\kappa n^{-1-a}}{s-s_{i}^{n}}+\frac{1}{2 n}|x|^{2} .
\end{aligned}
$$

By the standard change of variable in the Crandall-Lions theory of viscosity solutions, we deduce from Corollaries 6.1 and 6.2 that the functions $u^{n, \lambda_{i}^{n}, \kappa}$ and $v^{n, \lambda_{i}^{n}, \kappa}$ are respectively viscosity subsolution and super-solution on $\left(s_{i}^{n}, s_{i+1}^{n}\right] \times \mathbb{R}^{d}$ of

$$
\begin{aligned}
& -\partial_{s} u^{n, \lambda_{i}^{n}, \kappa}-\bar{G}\left(s, \eta^{\lambda_{i}^{n}}(x), u^{n, \lambda_{i}^{n}, \kappa}, D u^{n, \lambda_{i}^{n}, \kappa}+\frac{x}{n}, D^{2} u^{n, \lambda_{i}^{n}, \kappa}+\frac{1}{n} \mathbb{I}_{d}\right)-R^{u, n}(x) \leq 0, \\
& -\partial_{s} v^{n, \lambda_{i}^{n}, \kappa}-\bar{G}\left(s, \eta^{\lambda_{i}^{n}}(x), v^{n, \lambda_{i}^{n}, \kappa}, D v^{n, \lambda_{i}^{n}, \kappa}-\frac{x}{n}, D^{2} v^{n, \lambda_{i}^{n}, \kappa}-\frac{1}{n} \mathbb{I}_{d}\right)+R^{v, n}(x) \geq 0,
\end{aligned}
$$

where $\bar{G}(\theta, y, z, \gamma)=-2 L_{0} y+e^{2 L_{0} t} G\left(\theta, e^{-2 L_{0} t} y, e^{-2 L_{0} t} z, e^{-2 L_{0} t} \gamma\right)$. In particular, note that

$$
\bar{G}(\theta, y, z, \gamma)-\bar{G}\left(\theta, y^{\prime}, z, \gamma\right) \leq-L_{0}\left(y-y^{\prime}\right)^{+}+3 L_{0}\left(y-y^{\prime}\right)^{-},
$$

and therefore

$$
L_{0}\left(y-y^{\prime}\right) \leq\left(\bar{G}\left(\theta, y^{\prime}, z, \gamma\right)-\bar{G}(\theta, y, z, \gamma)\right)^{+} \leq\left|\bar{G}\left(\theta, y^{\prime}, z, \gamma\right)-\bar{G}(\theta, y, z, \gamma)\right| .
$$

Proof of Theorem 4.2 Without loss of generality, we only prove $(u-v)(\mathbf{0}) \leq 0$.

1. Following the classical argument of doubling variables, for fixed $n$ and $i$, we define

$$
w^{\kappa, \varepsilon}\left(s, x, x^{\prime}\right):=u^{n, \lambda_{i}^{n}, \kappa}(s, x)-v^{n, \lambda_{i}^{n}, \kappa}\left(s, x^{\prime}\right)-\frac{1}{2 \varepsilon}\left|x-x^{\prime}\right|^{2} .
$$

There is a constant $C>0$ only dependent on $T$ and the bound of $u, v$, and a point $\left(\hat{s}_{\kappa, \varepsilon}, \hat{x}_{\kappa, \varepsilon}, \hat{x}_{\kappa, \varepsilon}^{\prime}\right) \in$ $Q_{n}:=\left[s_{i}^{n}+n^{-1-a} \kappa / C, s_{i+1}^{n}\right] \times O_{C \sqrt{n}} \times O_{C \sqrt{n}}$ such that

$$
w^{\kappa, \varepsilon}\left(\hat{s}_{\kappa, \varepsilon}, \hat{x}_{\kappa, \varepsilon}, \hat{x}_{\kappa, \varepsilon}^{\prime}\right)=\max _{\left(s, x, x^{\prime}\right) \in\left(s_{i}^{n}, s_{i+1}^{n}\right] \times \mathbb{R}^{d} \times \mathbb{R}^{d}} w^{\kappa, \varepsilon}\left(s, x, x^{\prime}\right) .
$$

Since $Q_{n}$ is compact, $\left\{\left(\hat{s}_{\kappa, \varepsilon}, \hat{x}_{\kappa, \varepsilon}, \hat{x}_{\kappa, \varepsilon}^{\prime}\right)\right\}_{\varepsilon}$ has a converging sub-sequence whose limit is denoted by $\left(\hat{s}_{\kappa}, \hat{x}_{\kappa}, \hat{x}_{\kappa}^{\prime}\right)$. In particular, it is easy to show that $\hat{x}_{\kappa}=\hat{x}_{\kappa}^{\prime}$.

2. We continue by discussing two alternative cases.

Case 1. Suppose that there are only a finite number of $\kappa$ such that $\hat{s}_{\kappa}<s_{i+1}^{n}$, and thus there is 
sub-sequence still denoted as $\left\{\hat{s}_{\kappa}\right\}_{\kappa}$ such that $\hat{s}_{\kappa} \equiv s_{i+1}^{n}$. By Corollaries 6.1 (ii) and 6.2 (ii), $u^{n, \lambda_{i}^{n}, \kappa}$ and $v^{n, \lambda_{i}^{n}, \kappa}$ are continuous on $\left(s_{i}^{n}, s_{i+1}^{n}\right]$. This provides for all $s \in\left(s_{i}^{n}, s_{i+1}^{n}\right]$ that

$$
\begin{aligned}
\left(u^{n, \lambda_{i}^{n}, \kappa}-v^{n, \lambda_{i}^{n}, \kappa}\right)(s, 0) & \leq \lim _{\varepsilon \rightarrow 0}\left\{u^{n, \lambda_{i}^{n}, \kappa}\left(\hat{s}_{\kappa, \varepsilon}, \hat{x}_{\kappa, \varepsilon}\right)-v^{n, \lambda_{i}^{n}, \kappa}\left(\hat{s}_{\kappa, \varepsilon}, \hat{x}_{\kappa, \varepsilon}^{\prime}\right)\right\} \\
& \leq\left(u^{n, \lambda_{i}^{n}, \kappa}-v^{n, \lambda_{i}^{n}, \kappa}\right)\left(s_{i+1}^{n}, \hat{x}_{\kappa}\right) \\
& \leq \sup _{|x| \leq C \sqrt{n}}\left(u^{n, \lambda_{i}^{n}, \kappa}-v^{n, \lambda_{i}^{n}, \kappa}\right)\left(s_{i+1}^{n}, x\right) \\
& \leq e^{2 L_{0} s_{i+1}^{n}} \sup _{|x| \leq C \sqrt{n}}\left(u^{n, \lambda_{i}^{n}}-v^{n, \lambda_{i}^{n}}\right)\left(s_{i+1}^{n}, x\right)
\end{aligned}
$$

We next send $\kappa \searrow 0$ and then $s \searrow s_{i}^{n}$. By the semicontinuity properties of $u^{n, \lambda_{i}^{n}}$ and $v^{n, \lambda_{i}^{n}}$ at $s_{i}^{n}$ stated in Corollaries 6.1 (iii) and 6.2 (iii), we obtain

$$
\left(u^{n, \lambda_{i}^{n}}-v^{n, \lambda_{i}^{n}}\right)\left(s_{i}^{n}, 0\right) \leq e^{\frac{2 L_{0} T}{n^{1+a}}} \sup _{|x| \leq C \sqrt{n}}\left(u^{n, \lambda_{i}^{n}}-v^{n, \lambda_{i}^{n}}\right)\left(s_{i+1}^{n}, x\right) .
$$

Case 2. Otherwise, there is a sub-sequence still denoted by $\left\{\hat{s}_{\kappa}\right\}_{\kappa}$ such that $\hat{s}_{\kappa}<s_{i+1}^{n}$ for each $\kappa$. Then, by the Crandall-Ishii Lemma in the parabolic case (see for example Theorem 12.2 on page 38 in [4), there are $\alpha, X, Y$ such that

$$
\left(\alpha, \varepsilon^{-1}\left(\hat{x}_{\kappa, \varepsilon}-\hat{x}_{\kappa, \varepsilon}^{\prime}\right), X\right) \in \underline{J} u^{n, \lambda_{i}^{n}, \kappa}\left(\hat{s}_{\kappa, \varepsilon}, \hat{x}_{\kappa, \varepsilon}\right), \quad\left(\alpha, \varepsilon^{-1}\left(\hat{x}_{\kappa, \varepsilon}-\hat{x}_{\kappa, \varepsilon}^{\prime}\right), Y\right) \in \bar{J} v^{n, \lambda_{i}^{n}, \kappa}\left(\hat{s}_{\kappa, \varepsilon}, \hat{x}_{\kappa, \varepsilon}^{\prime}\right),
$$

and $X \leq Y$. By the viscosity properties of $u^{n, \lambda_{i}^{n}, \kappa}$ and $v^{n, \lambda_{i}^{n}, \kappa}$ of Corollaries 6.1 a,d 6.2 respectively, this implies that

$$
\begin{aligned}
& -R^{u, n}\left(\hat{x}_{\kappa, \varepsilon}\right)-\bar{G}\left(\hat{s}_{\kappa, \varepsilon}, \eta^{\lambda_{i}^{n}}\left(\hat{x}_{\kappa, \varepsilon}\right), u^{n, \lambda_{i}^{n}, \kappa}, \varepsilon^{-1}\left(\hat{x}_{\kappa, \varepsilon}-\hat{x}_{\kappa, \varepsilon}^{\prime}\right)+\frac{\hat{x}^{\kappa, \varepsilon}}{n}, X+\frac{1}{n} \mathbb{I}_{d}\right) \\
& \leq 0 \leq R^{v, n}\left(\hat{x}_{\kappa, \varepsilon}^{\prime}\right)-\bar{G}\left(\hat{s}_{\kappa, \varepsilon}, \eta^{\lambda_{i}^{n}}\left(\hat{x}_{\kappa, \varepsilon}^{\prime}\right), v^{n, \lambda_{i}^{n}, \kappa}, \varepsilon^{-1}\left(\hat{x}_{\kappa, \varepsilon}-\hat{x}_{\kappa, \varepsilon}^{\prime}\right)-\frac{\hat{x}_{\kappa, \varepsilon}^{\prime}}{n}, Y-\frac{1}{n} \mathbb{I}_{d}\right) .
\end{aligned}
$$

By (6.1), we obtain

$$
\begin{aligned}
& L_{0}\left(u^{n, \lambda_{i}, \kappa}\left(\hat{s}_{\kappa, \varepsilon}, \hat{x}_{\kappa, \varepsilon}\right)-v^{n, \lambda_{i}, \kappa}\left(\hat{s}_{\kappa, \varepsilon}, \hat{x}_{\kappa, \varepsilon}^{\prime}\right)\right) \\
\leq & L_{0}\left(2 n^{-1}+n^{-1}\left|\hat{x}_{\kappa, \varepsilon}+\hat{x}_{\kappa, \varepsilon}^{\prime}\right|\right)+\rho^{G}\left(C\left|\hat{x}_{\kappa, \varepsilon}-\hat{x}_{\kappa, \varepsilon}^{\prime}\right|\right)+R^{u, n}\left(\hat{x}_{\kappa, \varepsilon}\right)+R^{v, n}\left(\hat{x}_{\kappa, \varepsilon}^{\prime}\right) \\
\leq & 2 C n^{-\frac{a}{p+1}}+2 L_{0}\left(n^{-1}+C n^{-\frac{1}{2}}\right)+\rho^{G}\left(C\left|\hat{x}_{\kappa, \varepsilon}-\hat{x}_{\kappa, \varepsilon}^{\prime}\right|\right)+\bar{\rho}\left(n^{-\frac{1}{p+1}}+i n^{-\frac{3 p+3}{2 p}}\left|x_{i}^{n}\right|_{p}\right),
\end{aligned}
$$

where $\bar{\rho}(\cdot):=\left(2 \rho^{G}+L_{0} \rho^{u}+L_{0} \rho^{v}\right)(C \cdot)$ and $\mathrm{x}_{i}^{n}:=\left(\mathrm{x}_{i-1}^{n}, x\right)$. Hence for any $s \in\left(s_{i}^{n}, s_{i+1}^{n}\right]$ we have

$$
\begin{aligned}
\left(u^{n, \lambda_{i}^{n}, \kappa}-v^{n, \lambda_{i}^{n}, \kappa}\right)(s, 0) & \leq \varlimsup_{\varepsilon \rightarrow 0}\left(u^{n, \lambda_{i}, \kappa}\left(\hat{s}_{\kappa, \varepsilon}, \hat{x}_{\kappa, \varepsilon}\right)-v^{n, \lambda_{i}, \kappa}\left(\hat{s}_{\kappa, \varepsilon}, \hat{x}_{\kappa, \varepsilon}^{\prime}\right)\right) \\
& \leq C n^{-\frac{a}{p+1}}+\bar{\rho}\left(n^{-\frac{1}{p+1}}+i n^{-\frac{3 p+3}{2 p}}\left|\mathrm{x}_{i}^{n}\right|_{p}\right) \\
& \leq C n^{-\frac{a}{p+1}}+\bar{\rho}\left(n^{-\frac{1}{p+1}}+i n^{-\frac{3 p+3}{2 p}}\left(\left|\mathrm{x}_{i-1}^{n}\right|_{p}+C \sqrt{n}\right)\right) .
\end{aligned}
$$

We next let $\kappa \searrow 0$ and then let $s \searrow s_{i}^{n}$. By the semicontinuity properties of $u^{n, \lambda_{i}^{n}}$ and $v^{n, \lambda_{i}^{n}}$ stated in Corollaries 6.1 (iii) and 6.2 (iii), we obtain

$$
\left(u^{n, \lambda_{i}^{n}}-v^{n, \lambda_{i}^{n}}\right)\left(s_{i}^{n}, 0\right) \leq C n^{-\frac{a}{p+1}}+\bar{\rho}\left(n^{-\frac{1}{p+1}}+i n^{-\frac{3 p+3}{2 p}}\left(\left|\mathrm{x}_{i-1}^{n}\right|_{p}+C \sqrt{n}\right)\right) .
$$


3. By the results of Cases 1 and 2 in the previous Step 2, we conclude that

$$
\begin{aligned}
\left(u^{n, \lambda_{i}^{n}}-v^{n, \lambda_{i}^{n}}\right)\left(s_{i}^{n}, 0\right) \leq \max \left\{e^{\frac{2 L_{0} T}{n^{1+a}}} \sup _{|x| \leq C \sqrt{n}}\left(u^{n, \lambda_{i}^{n}}-v^{n, \lambda_{i}^{n}}\right)\left(s_{i+1}^{n}, x\right),\right. \\
\\
\left.\quad C n^{-\frac{a}{p+1}}+\bar{\rho}\left(n^{-\frac{1}{p+1}}+i\left(\left|\mathrm{x}_{i-1}^{n}\right|_{p}+C \sqrt{n}\right) n^{-\frac{3 p+3}{2 p}}\right)\right\} .
\end{aligned}
$$

We next use Corollaries 6.1 (ii) and 6.2 (ii) so that by direct iteration, it follows that:

$$
\begin{array}{r}
\left(u^{n}-v^{n}\right)(\mathbf{0}) \leq e^{2 L_{0} T} \max \left\{\sup _{i \leq n^{1+a},\left|\mathrm{x}_{i}\right|_{p} \leq C n^{\hat{a}}}\left(u^{n}-v^{n}\right)\left(T, \eta^{\lambda_{i}}\left(x_{i}\right)\right),\right. \\
\left.C n^{-\frac{a}{p+1}}+\bar{\rho}\left(n^{-\frac{1}{p+1}}+C n^{1+a} n^{\hat{a}} n^{-\frac{3 p+3}{2 p}}\right)\right\}, \\
\leq e^{2 L_{0} T} \max \left\{\begin{array}{c}
\sup _{i \leq n^{1+a},\left|\mathrm{x}_{i}\right|_{p} \leq C n^{\hat{a}}}\left(u^{n}-v^{n}\right)\left(T, \eta^{\lambda_{i}}\left(x_{i}\right)\right), \\
\left.C n^{-\frac{a}{p+1}}+\bar{\rho}\left(n^{-\frac{1}{p+1}}+C n^{-\frac{1}{2 p}+a+\frac{a}{p}}\right)\right\},
\end{array}\right.
\end{array}
$$

where $\hat{a}:=\frac{1+a+p / 2}{p}=\frac{1}{2}+\frac{a+1}{p} \leq \frac{1}{2}+\frac{6}{5 p}$. Since $u(T, \cdot) \leq v(T, \cdot)$, we have

$$
\begin{aligned}
\left(u^{n}-v^{n}\right)(T, \eta) & \leq\left(u^{n}-u\right)(T, \eta)+(u-v)(T, \eta)-\left(v^{n}-v\right)(T, \eta) \\
& \leq\left|\left(u^{n}-u\right)(T, \eta)\right|+\left|\left(v^{n}-v\right)(T, \eta)\right|
\end{aligned}
$$

Recall the notation $\rho_{n}$ introduced in Lemma 5.5. Since $a<\frac{1}{5 p}$, we have

$$
\left(u^{n}-v^{n}\right)(\mathbf{0}) \leq e^{2 L_{0} T} \max \left\{2 \rho_{n}, C n^{-\frac{a}{p+1}}+\bar{\rho}\left(n^{-\frac{1}{p+1}}+C n^{-\frac{1}{10 p}}\right)\right\} .
$$

By Lemma [5.5. the regularizations $u^{n}$ and $v^{n}$ converge to $u$ and $v$, respectively. Then, by sending $n \rightarrow \infty$, we obtain the required result

$$
(u-v)(\mathbf{0}) \leq 0 .
$$

\section{Appendix}

In this section we provide sufficient conditions for the value function of a stochastic control problem to be $d_{p}$-uniformly continuous.

Example 7.1 Let $X$ be a controlled diffusion $d X_{s}^{\alpha}=\sigma\left(s, X^{\alpha}, \alpha_{s}\right) d W_{s}$, where $W$ is a Brownian motion, where the function $\sigma:(\theta, \alpha) \mapsto \sigma(\theta, \alpha)$ is bounded and $d_{p}$-Lipschitz continuous in $\theta$. Denote the shifted process:

$$
d X_{s}^{\alpha, \theta}=\sigma^{\theta}\left(s, X^{\alpha, \theta}, \alpha_{s}\right) d W_{s} .
$$

We consider the stochastic control problem:

$$
u_{0}:=\sup _{\|\alpha\|_{\infty} \leq 1} \mathbb{E}\left[g\left(X_{T \wedge}^{\alpha} \cdot\right)\right]
$$


where the function $g$ is uniformly continuous in $L^{p}$-norm, i.e. $\left|g(\omega)-g\left(\omega^{\prime}\right)\right| \leq \rho\left(\left\|\omega-\omega^{\prime}\right\|_{p}\right)$ (without loss of generality we may assume that $\rho$ is concave). Introduce the dynamic version:

$$
u(\theta):=\sup _{\|\alpha\|_{\infty} \leq 1} \mathbb{E}\left[g^{\theta}\left(X_{(T-t) \wedge}^{\alpha, \theta}\right)\right] .
$$

Our main objective in this section is to prove that

$$
\text { the function } u \text { is } d_{p} \text {-uniformly continuous. }
$$

To see this, we first estimate that

$$
\begin{aligned}
\left|u(t, \omega)-u\left(t, \omega^{\prime}\right)\right| & \leq \sup _{\alpha} \mathbb{E}\left|g^{t, \omega}\left(X_{(T-t) \wedge .}^{\alpha, t, \omega}\right)-g^{t, \omega^{\prime}}\left(X_{(T-t) \wedge .}^{\alpha, t, \omega^{\prime}}\right)\right| \\
& \leq \sup _{\alpha} \mathbb{E}\left[\rho\left(\left\|\omega_{t \wedge .}-\omega_{t \wedge}^{\prime} \cdot\right\|_{p}+\left\|X_{(T-t) \wedge}^{\alpha, t, \omega}-X_{(T-t) \wedge}^{\alpha, t, \omega^{\prime}}\right\|_{p}\right)\right] \\
& \leq \sup _{\alpha} \rho\left(d_{p}\left((t, \omega),\left(t, \omega^{\prime}\right)\right)+\mathbb{E}\left\|X_{(T-t) \wedge}^{\alpha, t, \omega}-X_{(T-t) \wedge}^{\alpha, t, \omega^{\prime}}\right\|_{p}\right),
\end{aligned}
$$

where we applied Jensen's inequality in the last step. We next focus on the estimation of

$$
\begin{aligned}
& \mathbb{E}\left\|X_{s \wedge(T-t) \wedge \cdot}^{\alpha, t, \omega}-X_{s \wedge(T-t) \wedge .}^{\alpha, t, \omega^{\prime}}\right\|_{p}^{2 p} \\
\leq & C \int_{0}^{T+1} \mathbb{E}\left|\int_{0}^{s \wedge(T-t) \wedge r}\left(\sigma^{t, \omega}\left(\lambda, X^{\alpha, t, \omega}, \alpha_{\lambda}\right)-\sigma^{t, \omega^{\prime}}\left(\lambda, X^{\alpha, t, \omega^{\prime}}, \alpha_{\lambda}\right)\right) d W_{\lambda}\right|^{2 p} d r \\
\leq & C \int_{0}^{T+1} \mathbb{E} \int_{0}^{s \wedge(T-t) \wedge r}\left|\sigma^{t, \omega}\left(\lambda, X^{\alpha, t, \omega}, \alpha_{\lambda}\right)-\sigma^{t, \omega^{\prime}}\left(\lambda, X^{\alpha, t, \omega^{\prime}}, \alpha_{\lambda}\right)\right|^{2 p} d \lambda d r \\
\leq & \left(2 C_{\text {lip }}\right)^{2 p} C(T+1) \int_{0}^{s}\left(d_{p}\left((t, \omega),\left(t, \omega^{\prime}\right)\right)^{2 p}+\mathbb{E}\left\|X_{\lambda \wedge(T-t) \wedge .}^{\alpha, t, \omega}-X_{\lambda \wedge(T-t) \wedge .}^{\alpha, t, \omega^{\prime}}\right\|_{p}^{2 p}\right) d \lambda,
\end{aligned}
$$

where $C_{\text {lip }}$ is the Lipschitz constant of $\sigma$. By the Gronwall inequality, this provides $\mathbb{E}\left\|X_{(T-t) \wedge}^{\alpha, t, \omega}-X_{(T-t) \wedge}^{\alpha, t, \omega^{\prime}}\right\|_{p}^{2 p} \leq \tilde{C} d_{p}\left((t, \omega),\left(t, \omega^{\prime}\right)\right)^{2 p}$, with $\tilde{C}=\left(2 C_{\text {lip }}\right)^{2 p} C(T+1) e^{\left(2 C_{\text {lip }}\right)^{2 p} C(T+1) T}$.

Plugging the last inequality into (7.2), we get

$$
\left|u(t, \omega)-u\left(t, \omega^{\prime}\right)\right| \leq \rho\left(\left(1+\tilde{C}^{\frac{1}{2 p}}\right) d_{p}\left((t, \omega),\left(t, \omega^{\prime}\right)\right)\right) .
$$

We next estimate $\left|u(t, \omega)-u\left(t^{\prime}, \omega\right)\right|$ for $t<t^{\prime}$. By the dynamic programming, we have

$$
\begin{aligned}
\left|u(t, \omega)-u\left(t^{\prime}, \omega\right)\right| & =\left|\sup _{\alpha} \mathbb{E}\left[u^{t, \omega}\left(t^{\prime}-t, X^{\alpha, t, \omega}\right)\right]-u\left(t^{\prime}, \omega\right)\right| \\
& \leq \sup _{\alpha} \mathbb{E}\left|u^{t, \omega}\left(t^{\prime}-t, X^{\alpha, t, \omega}\right)-u\left(t^{\prime}, \omega\right)\right| \\
& \leq \sup _{\alpha} \mathbb{E}\left[\rho\left(\left(1+\tilde{C}^{\frac{1}{2 p}}\right) d_{p}\left(\left(t^{\prime}, \omega \otimes_{t} X^{\alpha, t, \omega}\right),\left(t^{\prime}, \omega\right)\right)\right)\right] \\
& \leq \sup _{\alpha} \rho\left(\left(1+\tilde{C}^{\frac{1}{2 p}}\right) \mathbb{E}\left[d_{p}\left(\left(t^{\prime}, \omega \otimes_{t} X^{\alpha, t, \omega}\right),\left(t^{\prime}, \omega\right)\right)\right]\right) \\
& \left.\leq \sup _{\alpha} \rho\left(\left(1+\tilde{C}^{\frac{1}{2 p}}\right)\left(\int_{t}^{T+1} \mathbb{E}\left|X_{(s-t) \wedge\left(t^{\prime}-t\right)}^{\alpha, t, \omega}\right|^{p} d s\right)^{\frac{1}{p}}\right]\right),
\end{aligned}
$$

where we applied the result of (7.3) in the third inequality. Finally using the classical estimate

$$
\sup _{\alpha, t, \omega, r} \mathbb{E}\left|X_{r \wedge\left(t^{\prime}-t\right)}^{\alpha, t, \omega}\right|^{p} \leq \hat{C}\left(t^{\prime}-t\right)^{\frac{p}{2}}
$$


(because $\sigma$ is bounded), we see that

$$
\left|u(t, \omega)-u\left(t^{\prime}, \omega\right)\right| \leq \rho\left(\left(1+\tilde{C}^{\frac{1}{2 p}}\right)((T+1) \hat{C})^{\frac{1}{p}}\left(t^{\prime}-t\right)^{\frac{1}{2}}\right) .
$$

The required result (7.1) is now a direct consequence of (7.3) and (7.4).

\section{References}

[1] G. Barles and P. E. Souganidis, Convergence of approximation schemes for fully nonlinear second order equations. Asymptotic Analysis 4:271-283, 1991.

[2] L. A. Caffarelli and X. Cabre, Fully nonlinear elliptic equations. American Mathematical Society Colloquium Publications, 43. American Mathematical Society, Providence, RI, 1995.

[3] R. Cont and D. Fournie, Functional Itô calculus and stochastic integral representation of martingales, Annals of Probability, 41 (2013), 109-133.

[4] M. Bardi, M. G. Crandall, L. C. Evans, H. M. Sonner, and P. E. Souganidis, Viscosity Solutions and Applications, Lecture Notes in Mathematics 1660, Springer, 1995.

[5] M. G. Crandall, P.-L. Lions, Viscosity solutions of Hamilton-Jacobi equations. Trans. Amer. Math. Soc. 277 (1983), no. 1, 1-42.

[6] A. Cosso, F. Gozzi, M. Rosestolato, S. Federico, and N. Touzi, Path-dependent equations and viscosity solutions in infinite dimension. Preprint, arXiv:1502.05648.

[7] B. Dupire, Functional Itô calculus, papers.ssrn.com, (2009).

[8] I. Ekren, C. Keller, N. Touzi and J. Zhang, On viscosity solutions of path dependent PDEs. Ann. Probab. 42 (2014), no. 1, 204-236.

[9] I. Ekren, N. Touzi and J. Zhang, Optimal Stopping under Nonlinear Expectation, Stochastic Processes and Their Applications, 124 (2014), 3277-3311.

[10] I. Ekren, N. Touzi and J. Zhang, Viscosity Solutions of Fully Nonlinear Parabolic Path Dependent PDEs: Part I, Ann. Probab., to appear.

[11] I. Ekren, N. Touzi and J. Zhang, Viscosity Solutions of Fully Nonlinear Parabolic Path Dependent PDEs: Part II, Ann. Probab., to appear.

[12] R. Jensen, P.-L. Lions, and P. E. Souganidis, A uniqueness result for viscosity solutions of second order fully nonlinear partial differential equations, Proceedings of the American mathematical society, Vol. 102, No. 4, April 1988.

[13] J. M. Lasry and P.-L. Lions, A remark on regularization of Hilbert spaces, Israel J. Math., Vol. 55, No. 33, 1986. 
[14] N. Lukoyanov, On viscosity solution of functional Hamilton-Jacobi type equations for hereditary systems, Proceedings of the Steklov Institute of Mathematics, Suppl. 2, 190-200, 2007.

[15] S. Peng, Backward stochastic differential equation, nonlinear expectation and their applications, Proceedings of the International Congress of Mathematicians, Hyderabad, India, (2010).

[16] Peng, S. and Wang, F. (2011) BSDE, Path-dependent PDE and Nonlinear Feynman-Kac Formula, arXiv:1108.4317.

[17] T. Pham and J. Zhang, Two Person Zero-sum Game in Weak Formulation and Path Dependent Bellman-Isaacs Equation, SIAM Journal of Control and Optimization, 52 (2014), 2090-2121.

[18] Z. Ren, Perron's method for viscosity solutions of semilinear path dependent PDEs, preprint, arXiv:1503.02169

[19] Z. Ren, N. Touzi and J. Zhang, An overview on Viscosity Solutions of Path-Dependent PDEs, Stochastic Analysis and Applications 2014 - In Honour of Terry Lyons, Springer Proceedings in Mathematics and Statistics, Editors: Dan Crisan, Ben Hambly, Thaleia Zariphopoulou, Springer (2014), 397-453.

[20] Z. Ren and X. Tan, On the convergence of monotone schemes for path-dependent PDE. Preprint, arXiv:1504.01872.

[21] Z. Ren, N. Touzi and J. Zhang, Comparison of viscosity solutions of semilinear path-dependent partial differential equations. Preprint, arXiv:1410.7281,

[22] J. Zhang and J. Zhuo, Monotone Schemes for Fully Nonlinear Parabolic Path Dependent PDEs, Journal of Financial Engineering, 1 (2014) 1450005 (23 pages); DOI: $10.1142 / \mathrm{S} 2345768614500056$. 